\title{
Neuronal underpinnings of cognitive impairment and - improvement in mood disorders $\dagger$
}

\author{
Kamilla W. Miskowiak, " and Cecilia S. Petersen
}

Neurocognition and Emotion in Affective Disorder (NEAD) Group, Copenhagen Affective Disorder Research Centre, Copenhagen Psychiatric Centre, Copenhagen University Hospital, Copenhagen, Denmark; Department of Psychology, University of Copenhagen, Copenhagen, Denmark

Neuropsychiatric illnesses including mood disorders are accompanied by cognitive impairment, which impairs work capacity and quality of life. However, there is a lack of treatment options that would lead to solid and lasting improvement of cognition. This is partially due to the absence of valid and reliable neurocircuitry-based biomarkers for pro-cognitive effects. This systematic review therefore examined the most consistent neural underpinnings of cognitive impairment and cognitive improvement in unipolar and bipolar disorders. We identified 100 studies of the neuronal underpinnings of working memory and executive skills, learning and memory, attention, and implicit learning and 9 studies of the neuronal basis for cognitive improvements. Impairments across several cognitive domains were consistently accompanied by abnormal activity in dorsal prefrontal (PFC) cognitive control regions-with the direction of this activity depending on patients' performance levels-and failure to suppress default mode network (DMN) activity. Candidate cognition treatments seemed to enhance task-related dorsal PFC and temporo-parietal activity when performance increases were observed, and to reduce their activity when performance levels were unchanged. These treatments also attenuated DMN hyper-activity. In contrast, nonspecific cognitive improvement following symptom reduction was typically accompanied by decreased limbic reactivity and reversal of pre-treatment fronto-parietal hyperactivity. Together, the findings highlight some common neural correlates of cognitive impairments and cognitive improvements. Based on this evidence, studies are warranted to examine the reliability and predictive validity of target engagement in the identified neurocircuitries as a biomarker model of pro-cognitive effects.

Received 20 June 2017; Accepted 30 January 2018; First published online 10 September 2018

Key words: Cognitive enhancement, cognitive impairment, fMRI, mood disorders.

\section{Introduction}

Unipolar disorder (UD) and bipolar disorder (BD) are among the leading causes of disability worldwide. ${ }^{1}$ Common features of these mood disorders are persistent cognitive impairments across attention, memory, and executive function ${ }^{2,3}$ and profound socio-occupational disability. ${ }^{4-6}$ In particular, cognitive impairments directly contribute to patients' functional disability and high unemployment rates, ${ }^{4-6}$ which constitute the largest socio-economic costs of mood disorders. ${ }^{7,8}$

Notwithstanding the clear need for treatment to target patients' cognitive impairments, there are no

*Address for correspondence: Professor Kamilla W. Miskowiak, Psychiatric Centre Copenhagen, Copenhagen University Hospital, Rigshospitalet, Blegdamsvej 9, DK-2100 Copenhagen, Denmark. (Email: kamilla@miskowiak.dk)

† The Lundbeck Foundation and the Weimann Foundation are acknowledged for their contribution to KWM's salary from 2012-2020. clinically available treatments with direct pro-cognitive efficacy in mood disorders. ${ }^{9,10}$ Two recent systematic reviews of cognition trials revealed only preliminary evidence for potential efficacy of candidate treatments in UD and BD, respectively. ${ }^{9,10}$ The disappointing findings are likely to result from common methodological challenges across cognition trials in mood disorders. ${ }^{11}$ The International Society for Bipolar Disorder (ISBD) therefore convened an international task force to develop consensus-based recommendations for the design and methodology of cognition trials. ${ }^{11}$ One of the important task force recommendations was to include neuroimaging assessments in future cognition trials to explore treatment-related target engagement in the neurocircuitries underlying patients' cognitive impairments. Insights from such assessments may provide a platform for identification of a sensitive neurocircuitry-based biomarker model that can predict treatment efficacy on cognition and thus serve as a surrogate endpoint in treatment development programs. 
Converging evidence from preclinical studies and neuroimaging studies in mood disorders suggest that cognitive impairments arise from disruption of neuroplasticity mechanisms and associated functional and structural changes in cognition-relevant neurocircuitries. $^{12}$ Specifically, functional magnetic resonance imaging (fMRI) studies have documented aberrant encoding and working memory-related activity in the medial and dorsal prefrontal cortex (PFC), and temporal and parietal regions during acute mood episodes and remission. ${ }^{13-20}$ Patients' cognitive impairments may also be exacerbated by a failure to suppress neural activity in the default mode network (DMN), a network of medial brain regions implicated in self-referential thoughts and thought wandering. ${ }^{14,21}$ In keeping with these findings, emerging evidence from a few recent intervention trials in mood disorders indicates that cognitive improvements are accompanied by neural activity changes in similar fronto-parietal, temporal, and DMN networks. ${ }^{22-25}$ However, the precise location(s) and direction of the neural activity changes underlying cognitive impairments and cognitive improvements are unclear. The aims of the present systematic review were therefore to delineate (a) the most reliable neural underpinnings of cognitive impairments in mood disorders and (b) the emerging neural basis for direct or indirect cognitive improvements in response to candidate cognition treatments or reduction in mood symptoms, respectively.

\section{Methods}

\section{Search strategy}

The present systematic review followed the procedures of the Preferred Reporting Items for Systematic reviews and Meta-Analyses (PRISMA) statement. ${ }^{26}$ Systematic computerized searches were performed in the databases PubMed and PsycInfo from inception up until October 31, 2017 (see detailed search strategy in the Supplementary Material, available online). The title/abstract screening and subsequent full-text screening were performed by the authors. Disagreements were discussed and consensus reached in all cases.

\section{Selection criteria}

We included original research articles that examined the neural basis of cognitive impairment and/or improvement as measured with fMRI blood-oxygen-leveldependent (BOLD) techniques in adults with UD or BD irrespective of mood state. We excluded articles that (a) were not original (ie, meta-analyses and reviews), (b) were preclinical, (c) did not utilize the fMRI BOLD signal, (d) did not verify a diagnosis of UD or BD with either the Diagnostic and Statistical Manual of Mental
Disorders (DSM) ${ }^{27}$ or the International Classification of Diseases (ICD), ${ }^{28}$ and (e) examined pediatric/adolescent or geriatric populations. Articles were also excluded if (f) organic disease was present (including neurodegenerative diseases, brain tumors, head trauma, and brain surgery); (g) neuroimaging was not related to performance on an objective neuropsychological test; (h) they involved single-case reports; (i) they only relied on resting state fMRI; or (j) they examined the influence of genetic polymorphisms.

With regard to articles investigating cognitive impairment, we included studies with direct comparisons between UD or BD and healthy control (HC) groups, respectively. Regarding articles examining the neural underpinnings of cognitive improvement, we included trials assessing potential "direct" pro-cognitive effects on an intervention and those in which nonspecific cognitive improvement following symptom reduction was observed. In contrast, we excluded articles that (k) assessed cognitive side-effects of treatments. It was also an inclusion criterion for these trials that fMRI had been conducted both before and after treatment or, in the case of randomized controlled trials, at least after treatment completion.

\section{Results}

The systematic searches identified a total of 1362 unique articles after deletion of duplicates. An additional 9 references were identified from the reference lists of relevant reviews and meta-analyses. Based on the title/ abstract screening, 141 articles were included in the fulltext screening. Of these, 107 articles met the specified inclusion criteria and were included in the review. Figure 1 depicts the PRISMA flowchart of the screening procedure. The vast majority of studies $(\mathrm{k}=100)$ examined the neural correlates of cognitive impairments, while only 9 studies investigated the neural basis of treatment-related cognitive improvement. Studies of the neural underpinnings of cognitive impairments were grouped into the following cognitive domains based on the employed fMRI paradigms: "working memory," "executive skills," "learning and memory," "attention," and "implicit learning." When the neural correlates of more than one relevant fMRI paradigm was reported in the same article, the results for each paradigm was presented under their respective cognitive domain. Similarly, articles investigating the neural correlates of both cognitive impairment and cognitive improvement appear twice in the respective tables.

\section{Cognitive impairment}

The design and findings of the $100 \mathrm{fMRI}$ studies of cognitive impairments can be seen in Tables $1-3 .{ }^{13,14,16,18,20,24,29-122}$ 


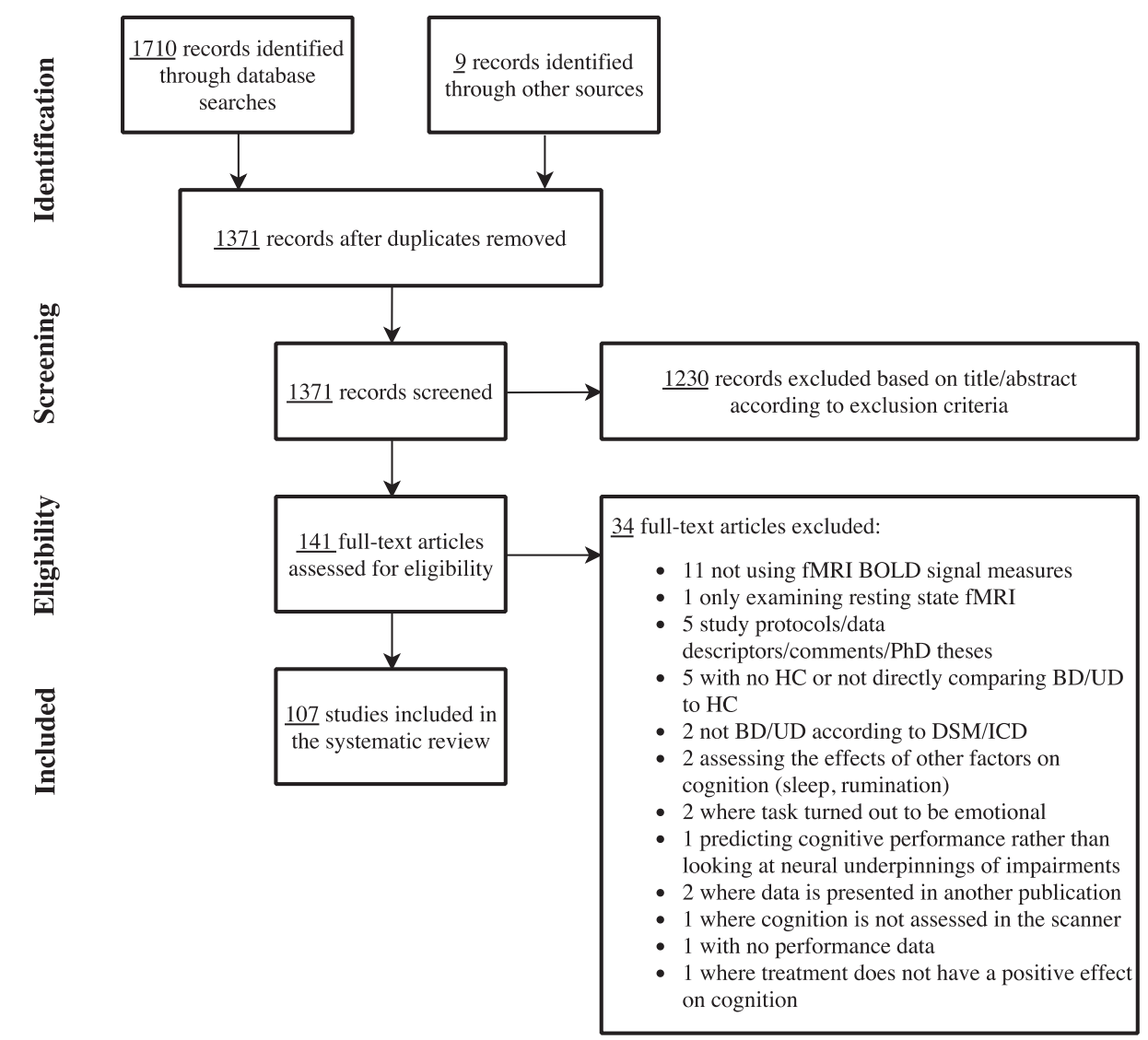

FIGURE 1. PRISMA flow chart. HC: Healthy control, BD: Bipolar disorder, UD: Unipolar disorder, DSM: Diagnostic and Statistical Manual of Mental Disorders (DSM), ${ }^{27}$ ICD: International Classification of Diseases (ICD). ${ }^{28}$

The studies were distributed across the domains "working memory" ( $\mathrm{k}=37$; Table 1$)$, "executive skills" $(\mathrm{k}=43$; Table 2), "learning and memory" (k=15; Table 3), "attention" ( $\mathrm{k}=6$; Table 3$)$, and "implicit learning" ( $\mathrm{k}=2$; Table 3$)$.

\section{Working memory}

Neural responses during working memory were assessed in 37 studies using a variety of fMRI paradigms, of which the most common ones were the n-back and delayed match-to-sample/Sternberg tasks. Twenty-two studies involved BD, 14 studies involved UD, and 1 study examined both groups (see Table 1).

Working memory in bipolar disorder. Thirteen (59\%) studies reported impaired working memory performance in symptomatic and remitted BD patients (ie, poorer accuracy and/or slowed response times), whereas 9 (41\%) studies found no performance impairment. The most robust findings were (i) hypo-activation in prefrontal cognitive control areas including the dorsolateral PFC (dlPFC) $)^{14,16,20,32,51,70,74,75}$ and (ii) failure to deactivate regions within the $\mathrm{DMN}$, most consistently the medial $\mathrm{PFC}^{32,33,55,113}$ in $\mathrm{BD}$ relative to $\mathrm{HC}$. These activity differences in cognitive control and DMN regions were commonly accompanied by impaired working memory performance. ${ }^{14,24,32,33,70,74,75,101,113}$ In keeping with this, a study specifically comparing cognitively impaired with cognitively intact $\mathrm{BD}$ patients revealed lower dlPFC activation in the impaired group. ${ }^{32}$ Another consistent finding was altered fronto-polar cortex activation in remitted $\mathrm{BD}$, with studies indicating increased activation at low task loads and decreased activation at high task load. ${ }^{29,45,63,101}$ Dorsolateral PFC hypo-activity thus seems to be linked to lower cognitive capacity (ie, impaired performance), while dlPFC hyper-activity may reflect lower cortical efficiency (ie, having to recruit more neural resources to maintain normal performance). Indeed, Adler $e t a l^{29}$ found that fMRI analyses co-varied for performance levels revealed task-related hyper-activation of fronto-polar cortex in remitted patients.

Finally, working memory performance in BD was also commonly associated with aberrant functional connectivity (FC) within subcortical and PFC structures as well as between subcortical structures and PFC, ${ }^{74,81,96,113}$ although the findings regarding the direction of these FC changes were heterogeneous. 
TABLE 1. Summary of included studies for the cognitive domain: working memory

\begin{tabular}{|c|c|c|c|c|}
\hline Author & Study design & Participants & fMRI paradigm & Findings \\
\hline $\begin{array}{l}\text { Gruber et al } \\
\qquad(2010)^{55}\end{array}$ & $\begin{array}{l}\text { Case-control } \\
\text { study }\end{array}$ & $\begin{array}{l}18 \text { euthymic BD } 1 \text { and } 18 \text { HC. } 83 \% \text { of BD medicated } \\
\text { (antidepressants, mood stabilizers, antipsychotics, } \\
\text { and/or benzodiazepines) }\end{array}$ & $\begin{array}{l}\text { Articulatory } \\
\quad \text { rehearsal task }\end{array}$ & $\begin{array}{l}\text { Performance: no difference. Activity: BD } \mathbf{T} \text { activation in right } \\
\text { amygdala and in the right frontal cortex (precentral gyrus), } \\
\text { intraparietal cortex, cerebellum, and frontal eye field. }\end{array}$ \\
\hline $\begin{array}{l}\text { McKenna et al } \\
\qquad(2014)^{74}\end{array}$ & $\begin{array}{l}\text { Case-control } \\
\text { study }\end{array}$ & $\begin{array}{l}23 \text { euthymic BD } 1 \text { and } 23 \text { HC. BD medicated } \\
\text { (antidepressants, mood stabilizers, antipsychotics, } \\
\text { and/or benzodiazepines) }\end{array}$ & $\begin{array}{l}\text { Delayed match-to- } \\
\text { sample task }\end{array}$ & $\begin{array}{l}\text { Performance: poorer in BD. Activity: Encoding: } \downarrow \text { in bilateral } \\
\text { medial PFC and right dIPFC, bilateral caudate/thalamus/ } \\
\text { insula, and left middle temporal gyrus. Maintenance: BD } \\
\uparrow \text { right postcentral gyrus, right middle occipital cortex/ } \\
\text { middle temporal cortex, and bilateral cuneus. } \\
\text { Connectivity: encoding: trend for diff. in functional } \\
\text { connectivity between bilateral medial PFC and right IFG: } \\
\text { medium effect size: BD } \uparrow \text { connectivity }><\text { HC. }\end{array}$ \\
\hline $\begin{array}{l}\text { Stegmayer } \\
\quad \text { et al } \\
(2015)^{96}\end{array}$ & $\begin{array}{l}\text { Case-control } \\
\text { study }\end{array}$ & $\begin{array}{l}18 \text { euthymic BD and } 18 \text { HC. } 83 \% \text { of BD medicated } \\
\text { (antidepressants, mood stabilizers, antipsychotics, } \\
\text { and/or benzodiazepines) }\end{array}$ & $\begin{array}{l}\text { Delayed match-to- } \\
\text { sample/ } \\
\text { Sternberg task }\end{array}$ & $\begin{array}{l}\text { Performance: trend toward poorer in BD in articulatory } \\
\text { rehearsal task. Activity: BD } \downarrow \text { negative functional } \\
\text { connectivity between right amygdala and right precentral } \\
\text { gyrus, right frontal eye field, and (pre)-SMA. }\end{array}$ \\
\hline $\begin{array}{l}\text { Monks et al } \\
\qquad(2004)^{18}\end{array}$ & $\begin{array}{l}\text { Case-control } \\
\text { study }\end{array}$ & $\begin{array}{l}12 \text { euthymic BD } 1 \text { and } 12 \text { HC. BD medicated (mood } \\
\text { stabilizers) }\end{array}$ & $\begin{array}{l}\text { Delayed match-to- } \\
\text { sample/ } \\
\text { Sternberg task }\end{array}$ & Performance: No difference. Activity: № difference. \\
\hline $\begin{array}{l}\text { Lagopoulos } \\
\quad \text { et al } \\
(2007)^{70}\end{array}$ & $\begin{array}{l}\text { Case-control } \\
\text { study }\end{array}$ & $\begin{array}{l}10 \text { euthymic } \mathrm{BD} \text { and } 10 \mathrm{HC} .70 \% \text { of BD medicated (mood } \\
\text { stabilizers) }\end{array}$ & $\begin{array}{l}\text { Delayed match-to- } \\
\text { sample/ } \\
\text { Sternberg task }\end{array}$ & $\begin{array}{l}\text { Performance: poorer in BD. Activity: Encoding: BD } \downarrow \text { activity } \\
\text { in right inferior frontal gyrus. Delay: BD } \downarrow \text { activity in the } \\
\text { right parahippocampal, inferior, and middle frontal gyri } \\
\text { and } \mathbf{T} \text { activity in medial frontal gyrus. Response } \\
\text { condition: BD } \downarrow \text { activity in the superior frontal and } \\
\text { anterior cingulate gyri. }\end{array}$ \\
\hline $\begin{array}{l}\text { Walter et al } \\
\qquad(2007)^{108}\end{array}$ & $\begin{array}{l}\text { Case-control } \\
\text { study }\end{array}$ & $\begin{array}{l}12 \text { partially remitted UD and } 17 \text { HC. All UD medicated } \\
\text { (antidepressants) }\end{array}$ & $\begin{array}{l}\text { Delayed match-to- } \\
\text { sample/ } \\
\text { Sternberg task }\end{array}$ & $\begin{array}{l}\text { Performance: poorer in UD for high cognitive loads. Activity: } \\
\text { UD } \uparrow \text { left dIPFC with highest cognitive load and } \uparrow \\
\text { activation in vmPFC during the control condition. Activity } \\
\text { during correct trials also } \uparrow \text { dIPFC in UD. }\end{array}$ \\
\hline $\begin{array}{l}\text { Vasic et al } \\
\qquad(2009)^{105}\end{array}$ & $\begin{array}{l}\text { Case-control } \\
\text { study }\end{array}$ & $\begin{array}{l}14 \text { depressed UD and } 14 \text { HC. All UD medicated } \\
\text { (antidepressants) }\end{array}$ & $\begin{array}{l}\text { Delayed match-to- } \\
\text { sample/ } \\
\text { Sternberg task }\end{array}$ & $\begin{array}{l}\text { Performance: poorer in UD, especially at high WM loads. } \\
\text { Activity: UD } \downarrow \text { functional connectivity in fronto-parietal } \\
\text { network including inferior parietal, superior prefrontal, } \\
\text { and frontopolar regions. UD } \uparrow \text { functional connectivity in } \\
\text { left dIPFC and bilateral cerebellum. In a temporally anti- } \\
\text { correlated network, UD } \downarrow \text { connectivity in the ACC, the } \\
\text { vIPFC, and superior prefrontal cortex. UD } \uparrow \text { connectivity in } \\
\text { bilateral superior temporal cortex, left cuneus, and right } \\
\text { lingual gyrus. }\end{array}$ \\
\hline $\begin{array}{l}\text { McKenna et al } \\
\qquad(2015)^{75}\end{array}$ & $\begin{array}{l}\text { Case-control } \\
\text { study }\end{array}$ & $\begin{array}{l}26 \text { euthymic BD and } 36 \text { HC. BD medicated } \\
\text { (antidepressants, mood stabilizers, antipsychotics, } \\
\text { and/or benzodiazepines) }\end{array}$ & $\begin{array}{l}\text { Delayed match-to- } \\
\text { sample task and } \\
\mathrm{N} \text {-task }\end{array}$ & $\begin{array}{l}\text { Performance: poorer in BD. Activity: BD: } \downarrow \text { left dIPFC activity } \\
\text { during encoding. Activity in bilateral dIPFC during } \\
\text { encoding predicted performance in BD. }\end{array}$ \\
\hline $\begin{array}{l}\text { Robinson et al } \\
\qquad(2009)^{88}\end{array}$ & $\begin{array}{l}\text { Case-control } \\
\text { study }\end{array}$ & $\begin{array}{l}15 \text { remitted BD and } 15 \text { HC. } 93 \% \text { of BD medicated } \\
\text { (antidepressants, mood stabilizers, and/or } \\
\text { antipsychotics) }\end{array}$ & $\begin{array}{l}\text { Delayed non-match- } \\
\text { to-sample task }\end{array}$ & $\begin{array}{l}\text { Performance: no difference. Activity: BD: } \mathbf{\uparrow} \text { retrieval-related } \\
\text { activation in frontal regions and } \downarrow \text { activation in parieto- } \\
\text { occipital and temporal lobes. Novelty condition (encoding } \\
\text { of new memories): BD } \downarrow \text { activation in occipital and } \\
\text { temporal lobe regions, posterior cingulate, left } \\
\text { parahippocampal gyrus, left cuneus, bilateral fusiform } \\
\text { gyrus and } \boldsymbol{\uparrow} \text { activity in right precentral gyrus, middle and } \\
\text { inferior frontal gyrus, left medial frontal gyrus, and ACC. }\end{array}$ \\
\hline $\begin{array}{l}\text { Le et al } \\
\qquad(2017)^{71}\end{array}$ & $\begin{array}{l}\text { Case-control } \\
\text { study }\end{array}$ & 18 unmedicated depressed UD and $21 \mathrm{HC}$ & $\begin{array}{l}\text { Delayed recognition } \\
\text { task }\end{array}$ & $\begin{array}{l}\text { Performance: poorer in UD. Activity: Retrieval scene }>\text { face } \\
\text { contrast: UD } \downarrow \text { activation in right and left transverse } \\
\text { occipital sulcus/middle occipital gyrus and left posterior } \\
\text { parietal. Memory-load effect weaker in UD. UD also } \\
\text { aberrant retrieval-related functional connectivity between } \\
\text { middle frontal gyrus and parahippocampal place area. }\end{array}$ \\
\hline $\begin{array}{l}\text { Townsend } \\
\qquad \text { et al } \\
(2010)^{20}\end{array}$ & $\begin{array}{l}\text { Case-control } \\
\text { study }\end{array}$ & $\begin{array}{l}42 \text { manic, euthymic or depressed BD and } 14 \text { HC. BD } \\
\text { medicated (antidepressants, mood stabilizers, and/or } \\
\text { antipsychotics) }\end{array}$ & N-back task & $\begin{array}{l}\text { Performance: no difference. Activity: BD } \downarrow \text { right dIPFC } \\
\text { activity, irrespective of mood state. BD } \downarrow \text { right parietal } \\
\text { activity, irrespective of mood state. }\end{array}$ \\
\hline $\begin{array}{l}\text { Wu et al } \\
\qquad(2014)^{113}\end{array}$ & $\begin{array}{l}\text { Case-control (3 } \\
\text { sample } \\
\text { design) }\end{array}$ & $\begin{array}{l}20 \text { BD-I in depression or remission and } 29 \mathrm{HC} \text { (and } 36 \\
\text { SZ). BD medicated (antidepressants, mood } \\
\text { stabilizers, antipsychotics, and/or benzodiazepines) }\end{array}$ & N-back task & $\begin{array}{l}\text { Performance: poorer in BD in 2-back task. Activity: BD } \uparrow \\
\text { activity in left posterior cingulate cortex and medial PFC in } \\
\text { 2-back task (less deactivation in BD }><H C \text { ). Aberrant } \\
\text { effective connectivity in BD: positive from left PCC to } \\
\text { mPFC and negative from mPFC to PCC. }\end{array}$ \\
\hline
\end{tabular}


TABLE 1. Continued

\begin{tabular}{|c|c|c|c|c|}
\hline Author & Study design & Participants & fMRI paradigm & Findings \\
\hline $\begin{array}{l}\text { Thermenos } \\
\qquad \text { et al } \\
(2010)^{101}\end{array}$ & $\begin{array}{l}\text { Case-control } \\
\text { study }\end{array}$ & $\begin{array}{l}19 \text { stable BD, } 18 \text { relatives, and } 19 \text { HC. BD medicated } \\
\text { (antidepressants, mood stabilizers, antipsychotics, } \\
\text { and/or benzodiazepines) }\end{array}$ & $\mathrm{N}$-back task & $\begin{array}{l}\text { Performance: poorer in BD. Activity: BD and relatives } \uparrow \\
\text { activation in left anterior insula (less deactivation). BD } \downarrow \\
\text { left frontopolar cortex activity }><H C \text { and relatives. }\end{array}$ \\
\hline $\begin{array}{l}\text { Adler et al } \\
\qquad(2004)^{29}\end{array}$ & $\begin{array}{l}\text { Case-control } \\
\text { study }\end{array}$ & $\begin{array}{l}15 \text { euthymic BD and } 15 \text { HC. } 67 \% \text { of BD medicated } \\
\text { (antidepressants, mood stabilizers, and/or } \\
\text { antipsychotics) }\end{array}$ & $\mathrm{N}$-back task & $\begin{array}{l}\text { Performance: poorer in BD. Activity: BD } \boldsymbol{\uparrow} \text { activation in fronto- } \\
\text { polar cortex, basal ganglia, thalamus, temporal cortex, } \\
\text { and posterior parietal cortex (when covarying for } \\
\text { performance) and } \downarrow \text { activation in posterior cingulate. }\end{array}$ \\
\hline $\begin{array}{l}\text { Brooks et al } \\
\qquad(2015)^{38}\end{array}$ & $\begin{array}{l}\text { Case-control } \\
\text { study }\end{array}$ & 19 depressed BD 2 and $19 \mathrm{HC}$. BD unmedicated & $\mathrm{N}$-back task & $\begin{array}{l}\text { Performance: no difference. Activity: BD } \downarrow \text { activation in left } \\
\text { middle frontal gyrus, left superior frontal gyrus, left } \\
\text { inferior parietal lobule, left middle temporal gyrus/angular } \\
\text { gyrus, and bilateral occipital regions. }\end{array}$ \\
\hline $\begin{array}{l}\text { Fernández- } \\
\text { Concuera } \\
\text { et al } \\
(2013)^{14}\end{array}$ & $\begin{array}{l}\text { Case-control } \\
\text { study }\end{array}$ & $\begin{array}{l}41 \text { depressed BD and } 41 \text { HC. BD medicated } \\
\text { (antidepressants, mood stabilizers, and/or } \\
\text { antipsychotics) }\end{array}$ & $\mathrm{N}$-back task & $\begin{array}{l}\text { Performance: Poorer in BD. Activity: BD } \downarrow \text { activation in } \\
\text { bilateral dIPFC and cerebellum. }\end{array}$ \\
\hline $\begin{array}{l}\text { Drapier et al } \\
\qquad(2008)^{45}\end{array}$ & $\begin{array}{l}\text { Case-control } \\
\text { study }\end{array}$ & $\begin{array}{l}20 \text { remitted BD 1, } 20 \text { relatives, and } 20 \text { HC. BD medicated } \\
\text { (antidepressants, mood stabilizers, and/or } \\
\text { antipsychotics) }\end{array}$ & $\mathrm{N}$-back task & $\begin{array}{l}\text { Performance: poorer in } \mathrm{BD} \text { ( }><\mathrm{HC} \text { and relatives). Activity: } \\
\mathrm{BD} \uparrow \text { activation during 1-back in frontal pole and in right } \\
\text { parietal lobe/precuneus } \uparrow \text { activation in right and left } \\
\text { parietal lobe/precuneus during 2-back. }\end{array}$ \\
\hline $\begin{array}{l}\text { Jogia et al } \\
\qquad(2012)^{63}\end{array}$ & $\begin{array}{l}\text { Case-control } \\
\text { study }\end{array}$ & $\begin{array}{l}36 \text { euthymic BD } 1 \text { and } 37 \text { HC. } 61 \% \text { of BD medicated } \\
\text { (antidepressants, mood stabilizers, and/or } \\
\text { antipsychotics) }\end{array}$ & $\mathrm{N}$-back task & $\begin{array}{l}\text { Performance: no difference. Activity: BD } \boldsymbol{\uparrow} \text { activation in right } \\
\text { superior and middle temporal gyri during high-load (3- } \\
\text { back), and in } \boldsymbol{\uparrow} \text { vIPFC during low to medium loads (0-2- } \\
\text { back) and } \downarrow \text { vIPFC activation during high load (3-back). }\end{array}$ \\
\hline $\begin{array}{l}\text { Palaniyappan } \\
\quad \text { et al } \\
\quad(2014)^{81}\end{array}$ & $\begin{array}{l}\text { Case-control } \\
\text { study }\end{array}$ & $\begin{array}{l}20 \text { stable, psychotic BD and } 34 \mathrm{HC} \text { (and } 39 \mathrm{SZ} \text { ). BD } \\
\text { medicated (antidepressants, mood stabilizers, and/or } \\
\text { antipsychotics) }\end{array}$ & N-back task & $\begin{array}{l}\text { Performance: poorer in BD. Activity: BD: } \mathbf{I} \text { degree of centrality } \\
\text { in the hippocampus/parahippocampus and in thalamic } \\
\text { regions and lateral parietal cortex and } \downarrow \text { connectivity of } \\
\text { the right insula. }\end{array}$ \\
\hline $\begin{array}{l}\text { Dell'0sso et al } \\
\quad(2015)^{42}\end{array}$ & $\begin{array}{l}\text { Case-control } \\
\text { study }\end{array}$ & $\begin{array}{l}28 \text { euthymic BD (15 BD 1, } 13 \text { BD 2) and } 27 \text { HC. BD } \\
\text { medicated (antidepressants, mood stabilizers, and/or } \\
\text { antipsychotics) }\end{array}$ & N-back task & $\begin{array}{l}\text { Performance: no difference. Activity: BD } \uparrow \text { right middle } \\
\text { frontal gyrus engagement regardless of WM load. } \\
\text { Especially, BD } 1 \text { had greater BOLD signal change, while } \\
\text { BD } 2 \text { expressed an intermediate pattern of activation. }\end{array}$ \\
\hline $\begin{array}{l}\text { Alonso-Lana } \\
\qquad \text { et al } \\
\qquad(2016)^{32}\end{array}$ & $\begin{array}{l}\text { Case-control (3 } \\
\text { sample } \\
\text { design) }\end{array}$ & $\begin{array}{l}50 \text { euthymic BD (27 cognitively preserved, } 23 \text { cognitively } \\
\text { impaired) and } 28 \text { HC. BD (both groups) medicated } \\
\text { (antidepressants, mood stabilizers, and/or } \\
\text { antipsychotics) }\end{array}$ & N-back task & $\begin{array}{l}\text { Performance: poorer in all patients }><\text { HC. Activity: } \\
\text { cognitively impaired patients } \downarrow \text { right dIPFC than } \\
\text { cognitively preserved patients during high-load WM. Both } \\
\text { patient groups } \uparrow \text { activation in medial frontal cortex } \\
\text { (failure to deactivate) }><\text { HC. }\end{array}$ \\
\hline $\begin{array}{l}\text { Alonso-Lana } \\
\quad \text { et al } \\
(2016)^{33}\end{array}$ & $\begin{array}{l}\text { Case-control (3 } \\
\text { sample } \\
\text { design) }\end{array}$ & $\begin{array}{l}20 \text { euthymic BD, } 20 \text { relatives, and } 40 \mathrm{HC} \text {. BD medicated } \\
\text { (antidepressants, mood stabilizers, and/or } \\
\text { antipsychotics) }\end{array}$ & N-back task & $\begin{array}{l}\text { Performance: poorer in BD. Activity: BD (and relatives) } \uparrow \\
\text { activity (failure to deactivate) cluster in medial PFC } \\
\text { during WM (less marked in relatives). }\end{array}$ \\
\hline $\begin{array}{l}\text { Monks et al } \\
\qquad(2004)^{18}\end{array}$ & $\begin{array}{l}\text { Case-control } \\
\text { study }\end{array}$ & $\begin{array}{l}12 \text { euthymic BD } 1 \text { and } 12 \text { HC. BD medicated (mood } \\
\text { stabilizers) }\end{array}$ & $\mathrm{N}$-back task & $\begin{array}{l}\text { Performance: No difference. Activity: BD } \downarrow \text { bilateral frontal, } \\
\text { temporal, and parietal activation (anterior cingulate } \\
\text { gyrus, right medial frontal and middle temporal gyrus and } \\
\text { bilaterally in the inferior frontal gyrus, middle frontal } \\
\text { gyrus, precuneus, and cerebellar regions). BD } \boldsymbol{\uparrow} \text { activity in } \\
\text { left precentral, right medial frontal and left } \\
\text { supramarginal gyri. }\end{array}$ \\
\hline $\begin{array}{l}\text { Brandt et al } \\
\qquad(2014)^{37}\end{array}$ & $\begin{array}{l}\text { Case-control } \\
\text { study }\end{array}$ & $\begin{array}{l}100 \text { euthymic, psychotic, elevated or depressed BD and } \\
100 \mathrm{HC} \text { (and } 100 \mathrm{SZ} \text { ). BD medicated } \\
\text { (antidepressants, mood stabilizers, antipsychotics, } \\
\text { and/or benzodiazepines) }\end{array}$ & $\mathrm{N}$-back task & $\begin{array}{l}\text { Performance: poorer in } \mathrm{BD} \text {. Activity: no difference in any } \\
\text { components in } \mathrm{BD}><\mathrm{HC} \text {. }\end{array}$ \\
\hline $\begin{array}{l}\text { Frangou } \\
\qquad(2005)^{51}\end{array}$ & $\begin{array}{l}\text { Case-control } \\
\text { study }\end{array}$ & $\begin{array}{l}7 \text { remitted BD } 1 \text { and } 7 \text { HC (43 of each in the study but } \\
\text { only a subsample undergoes fMRI). BD medicated } \\
\text { (antidepressants, mood stabilizers, and/or } \\
\text { antipsychotics) }\end{array}$ & $\mathrm{N}$-back task & $\begin{array}{l}\text { Performance: no difference. Activity: BD } \downarrow \text { activity in dorsal } \\
\text { PFC and anterior cingulate and } \mathbf{T} \text { in another part of } \\
\text { superior frontal PFC during high memory demands. }\end{array}$ \\
\hline $\begin{array}{l}\text { Fitzgerald } \\
\qquad \text { et al } \\
\qquad(2008)^{48}\end{array}$ & $\begin{array}{l}\text { Case-control } \\
\text { study }\end{array}$ & $\begin{array}{l}13 \text { depressed UD and } 13 \text { HC. } 85 \% \text { of UD medicated } \\
\text { (antidepressants) }\end{array}$ & $\mathrm{N}$-back task & $\begin{array}{l}\text { Performance: no difference. Activity: UD } \uparrow \text { bilateral activity in } \\
\text { middle, medial, inferior frontal gyri, anterior cingulate } \\
\text { gyrus, precentral gyrus, inferior parietal lobule, superior } \\
\text { temporal gyrus, cuneus/precuneus, and thalamus. UD } \uparrow \\
\text { right orbital gyrus and left middle temporal gyrus. }\end{array}$ \\
\hline $\begin{array}{l}\text { Bartova et al } \\
\qquad(2015)^{35}\end{array}$ & $\begin{array}{l}\text { Case-control } \\
\text { study }\end{array}$ & 78 remitted UD and $42 \mathrm{HC}$. UD unmedicated & $\mathrm{N}$-back task & $\begin{array}{l}\text { Performance: no difference. Activity: UD } \uparrow \text { DMN activity (less } \\
\text { deactivation) during WM; strongest differences in the }\end{array}$ \\
\hline
\end{tabular}


TABLE 1. Continued

\begin{tabular}{|c|c|c|c|c|}
\hline Author & Study design & Participants & fMRI paradigm & Findings \\
\hline & & & & $\begin{array}{l}\text { anterior-medial (am) PFC. Activation patterns of adult- } \\
\text { onset UD did not sig. differ from HC. Adolescent-onset UD } \\
\text { ( }><\text { HC) } \downarrow \text { functional coupling between amPFC and } \\
\text { medial, middle, and superior frontal gyrus and precuneus } \\
\text { and } \mathbf{\uparrow} \text { amPFC-dIPFC coupling. Adult-onset UD: } \\
\text { qualitatively similar, but less-pronounced. }\end{array}$ \\
\hline $\begin{array}{l}\text { Garrett et al } \\
\qquad(2011)^{52}\end{array}$ & $\begin{array}{l}\text { Case-control } \\
\text { study }\end{array}$ & $\begin{array}{l}16 \text { depressed, psychotic UD, } 16 \text { depressed, nonpsychotic } \\
\text { UD, and } 19 \text { HC. UD medicated (antidepressants, mood } \\
\text { stabilizers, antipsychotics and/or benzodiazepines) }\end{array}$ & N-back task & 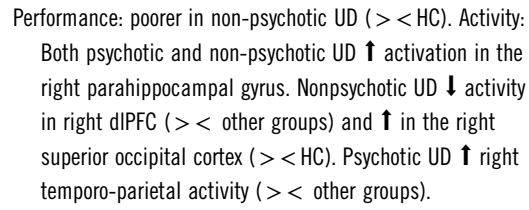 \\
\hline $\begin{array}{l}\text { Matsuo et al } \\
\qquad(2007)^{73}\end{array}$ & $\begin{array}{l}\text { Case-control } \\
\text { study }\end{array}$ & $\begin{array}{l}15 \text { depressed UD and } 15 \text { HC (only performance data for } \\
10 \text { HC, } 9 \text { UD). UD unmedicated }\end{array}$ & $\mathrm{N}$-back task & $\begin{array}{l}\text { Performance: no difference. Activity: UD } \uparrow \text { left dIPFC, middle } \\
\text { and superior frontal gyrus activation }(><\mathrm{HC}) \text {. }\end{array}$ \\
\hline $\begin{array}{l}\text { Norbury et al } \\
\qquad(2014)^{78}\end{array}$ & $\begin{array}{l}\text { Case-control } \\
\text { study }\end{array}$ & 15 remitted UD and 15 HC. UD unmedicated & $\mathrm{N}$-back task & $\begin{array}{l}\text { Performance: no difference. Activity: Quadratic load: UD } \\
\text { showed a positive quadratic load response in the bilateral } \\
\text { hippocampus; the converse was true for HC. UD } \boldsymbol{\uparrow} \text { activity } \\
\text { in bilateral hippocampus (less deactivation }><\mathrm{HC} \text { ). }\end{array}$ \\
\hline $\begin{array}{l}\text { Rodriguez- } \\
\text { Cano et al } \\
\quad(2014)^{89}\end{array}$ & $\begin{array}{l}\text { Case-control } \\
\text { study }\end{array}$ & $\begin{array}{l}26 \text { depressed UD and } 52 \text { HC. UD medicated } \\
\text { (antidepressants, mood stabilizers, and/or } \\
\text { antipsychotics) }\end{array}$ & $\mathrm{N}$-back task & $\begin{array}{l}\text { Performance: poorer in UD. Activity: 2-back vs. baseline: UD } \downarrow \\
\text { activation in the left dIPFC, extending to the precentral } \\
\text { gyrus and the frontal operculum, also incl. left and right } \\
\text { thalamus and left caudate. 2) cluster in the precuneus, } \\
\text { reaching the cuneus and bilateral superior parietal cortex } \\
3+4 \text { ) clusters in the cerebellum which also reached the } \\
\text { left inferior occipital cortex. UD } \uparrow \text { medial frontal cortex } \\
\text { and perigenual anterior cingulate cortex (reduced } \\
\text { deactivation }><\mathrm{HC} \text { ). }\end{array}$ \\
\hline $\begin{array}{l}\text { Schöning et al } \\
\quad(2009)^{94}\end{array}$ & $\begin{array}{l}\text { Case-control } \\
\text { study }\end{array}$ & $\begin{array}{l}28 \text { euthymic UD and } 28 \text { HC. UD medicated } \\
\text { (antidepressants) }\end{array}$ & $\mathrm{N}$-back task & $\begin{array}{l}\text { Performance: no difference. Activity: UD } \mathbf{\uparrow} \text { cingulate cortex } \\
\text { activity; dIPFC and vIPFC activation comparable between } \\
\text { UD and HC. }\end{array}$ \\
\hline $\begin{array}{l}\text { Barch et al } \\
\qquad(2003)^{34}\end{array}$ & $\begin{array}{l}\text { Case-control } \\
\text { study }\end{array}$ & $\begin{array}{l}14 \text { depressed UD and } 49 \mathrm{HC} \text { (and } 38 \mathrm{SZ} \text { ). Only 14\% of UD } \\
\text { patients were medicated (antidepressants) }\end{array}$ & $\mathrm{N}$-back task & $\begin{array}{l}\text { Performance: no difference. Activity: UD } \downarrow \text { activity in bilateral } \\
\text { thalamus, right precentral gyrus, and right parietal cortex. }\end{array}$ \\
\hline $\begin{array}{l}\text { Rose et al } \\
\qquad(2006)^{90}\end{array}$ & $\begin{array}{l}\text { Case-control } \\
\text { study }\end{array}$ & $\begin{array}{l}9 \text { symptomatic UD patients on antidepressant } \\
\text { medication and } 9 \mathrm{HC}\end{array}$ & $\mathrm{N}$-back task & $\begin{array}{l}\text { Performance: no difference. Activity: UD } \mathbf{T} \text { activity in medial } \\
\text { orbitofrontal cortex/rostral ACC. }\end{array}$ \\
\hline $\begin{array}{l}\text { Harvey et al } \\
\qquad(2005)^{60}\end{array}$ & $\begin{array}{l}\text { Case-control } \\
\text { study }\end{array}$ & $\begin{array}{l}10 \text { moderately to severely depressed UD and } 10 \text { HC. UD } \\
\text { medicated (antidepressants and/or benzodiazepines). } \\
\text { All tested within the first } 2 \text { weeks of the } \\
\text { antidepressant treatment. }\end{array}$ & N-back task & $\begin{array}{l}\text { Performance: no difference. Activity: UD } \boldsymbol{\uparrow} \text { activation of the } \\
\text { left inferior and middle frontal gyrus and the dorsal } \\
\text { anterior cingulate. }\end{array}$ \\
\hline $\begin{array}{l}\text { Hammar et al } \\
\quad(2016)^{59}\end{array}$ & $\begin{array}{l}\text { Case-control } \\
\text { study }\end{array}$ & $\begin{array}{l}17 \text { remitted or partially remitted UD and } 17 \mathrm{HC} \text {. UD } \\
\text { medicated (not specified) }\end{array}$ & $\mathrm{N}$-back task & $\begin{array}{l}\text { Performance: poorer in UD. Activity: no diff. in activation in } \\
\text { ACC or dIPFC. UD } \downarrow \text { caudate and putamen. No correlations } \\
\text { between brain responses and performance. }\end{array}$ \\
\hline $\begin{array}{l}\text { Meusel et al } \\
\qquad(2013)^{24}\end{array}$ & $\begin{array}{l}\text { Case-control } \\
\text { study, } \\
\text { nonrando- } \\
\text { mized } \\
\text { clinical trial }\end{array}$ & $\begin{array}{l}35 \text { partially remitted BD/UD and } 15 \mathrm{HC} \text {. BD/UD } \\
\text { medicated (antidepressants, mood stabilizers, } \\
\text { antipsychotics, and/or benzodiazepines) }\end{array}$ & $\mathrm{N}$-back task & $\begin{array}{l}\text { Performance: poorer in UD/BD. Activity: patients } \downarrow \text { activation } \\
\text { in superior frontal gyrus during 2-back vs. 0-back } \\
\text { conditions. }\end{array}$ \\
\hline $\begin{array}{l}\text { Hamilton et al } \\
\qquad(2009)^{16}\end{array}$ & $\begin{array}{l}\text { Case-control } \\
\text { study }\end{array}$ & $\begin{array}{l}21 \text { euthymic BD } 1 \text { and } 38 \mathrm{HC} \text { (and } 20 \mathrm{SZ} \text { ). } 81 \% \text { of BD } \\
\text { patients medicated (antidepressants, mood } \\
\text { stabilizers, and/or antipsychotics) }\end{array}$ & $\begin{array}{l}\text { WM fMRI paradigm } \\
\text { from the } \\
\text { Functional } \\
\text { Reference } \\
\text { Battery }\end{array}$ & $\begin{array}{l}\text { Performance: no difference. Activity: BD } \downarrow \text { activity in occipital } \\
\text { regions incl. right primary visual cortex. }\end{array}$ \\
\hline
\end{tabular}

Abbreviations: HC: Healthy control, BD: Bipolar disorder, UD: Unipolar disorder, SZ: Schizophrenia, PFC: prefrontal cortex, mPFC: medical PFC, IPFC: lateral PFC, dIPFC: dorsolateral PFC, vmPFC: Ventromedial PFC, amPFC: Anterior medial PFC, SMA: Supplementary motor area, PCC: posterior cingulate cortex, STG: Superior temporal gyrus, WM: Working memory, $><$ : Compared to.

* The authors combined the results from the two fMRI tasks for an overall working memory score. 
TABLE 2. Summary of included studies for the cognitive domain: executive skills

\begin{tabular}{|c|c|c|c|c|}
\hline Author & Study design & Participants & fMRI paradigm & Findings \\
\hline Frangou $(2011)^{50}$ & $\begin{array}{l}\text { Case-control } \\
\text { study }\end{array}$ & $\begin{array}{l}46 \text { euthymic BD 1, } 48 \text { relatives, and } 71 \text { HC. BD } \\
\text { medicated (antidepressants, mood stabilizers, } \\
\text { and/or antipsychotics) }\end{array}$ & Color-word Stroop task & $\begin{array}{l}\text { Performance: no difference. Activity: BD } \downarrow \text { activation in } \\
\text { superior parietal lobule, the inferior parietal lobule, the } \\
\text { head of the caudate, and the vIPFC. BD: } \downarrow \text { functional } \\
\text { connectivity between the vIPFC, ACC, and insula. }\end{array}$ \\
\hline Pompei et al $(2011)^{84}$ & $\begin{array}{l}\text { Case-control } \\
\text { study }\end{array}$ & $\begin{array}{l}39 \text { euthymic BD 1, } 39 \text { relatives ( } 25 \text { healthy, } 14 \\
\text { UD), and } 48 \text { HC. BD medicated } \\
\text { (antidepressants, mood stabilizers, and/or } \\
\text { antipsychotics). UD relatives unmedicated }\end{array}$ & Color-word Stroop task & $\begin{array}{l}\text { Performance: no difference. Activity: BD and relatives } \\
\text { ( }><\text { HC): } \downarrow \text { response in posterior and inferior } \\
\text { parietal lobules (no difference. between relatives and } \\
\text { BD). Left caudate mean activation } \downarrow \text { in BD }><\text { HC } \\
\text { and relatives without Axis I diagnosis. Right inferior } \\
\text { frontal gyrus mean level activation } \downarrow \text { in BD }><\text { all } \\
\text { other groups incl. UD relatives. }\end{array}$ \\
\hline Kronhaus et al $(2006)^{69}$ & $\begin{array}{l}\text { Case-control } \\
\text { study }\end{array}$ & $\begin{array}{l}10 \text { euthymic BD (some with residual depression) } \\
\text { and } 11 \text { HC. BD medicated (antidepressants, } \\
\text { mood stabilizers, and/or antipsychotics) }\end{array}$ & Color-word Stroop task & $\begin{array}{l}\text { Performance: no difference. Activity: BD } \downarrow \text { activation of } \\
\text { visual and left dl- and vIPFC areas. BD } \downarrow \text { left orbital } \\
\text { and medial prefrontal cortices. }\end{array}$ \\
\hline Blumberg et al (2003) ${ }^{36}$ & $\begin{array}{l}\text { Case-control } \\
\text { study }\end{array}$ & $\begin{array}{l}36 \text { elevated, depressed, or euthymic BD } 1 \text { and } 20 \\
\text { HC. BD medicated (antidepressants, mood } \\
\text { stabilizers, antipsychotics, and/or } \\
\text { benzodiazepines). }\end{array}$ & Color-word Stroop task & $\begin{array}{l}\text { Performance: no difference. Activity: BD } \downarrow \text { activation in } \\
\text { left rostral ventral PFC independent of mood state. } \\
\text { Right ventral PFC: } \downarrow \text { increase in elevated mood }>< \\
\text { euthymic, left ventral PFC: } \mathbb{T} \text { increase in signal in } \\
\text { depressed group }><\text { euthymic group. }\end{array}$ \\
\hline Gruber et al $(2004)^{57}$ & $\begin{array}{l}\text { Case-control } \\
\text { study }\end{array}$ & $\begin{array}{l}11 \text { stable BD and } 10 \text { HC. BD medicated } \\
\text { (antidepressants, mood stabilizers, and/or } \\
\text { antipsychotics) }\end{array}$ & Color-word Stroop task & $\begin{array}{l}\text { Performance: interference task poorer in BD. Activity: } \\
\text { interference task: BD: } \downarrow \text { signal intensity in right } \\
\text { anterior cingulate (attention to action area). BD: } \boldsymbol{\uparrow} \\
\text { dIPFC activation. }\end{array}$ \\
\hline Pompei et al $(2011)^{83}$ & $\begin{array}{l}\text { Case-control } \\
\text { study }\end{array}$ & $\begin{array}{l}39 \text { euthymic BD 1, } 39 \text { relatives (25 healthy, } 14 \\
\text { UD), and } 48 \text { HC. BD medicated } \\
\text { (antidepressants, mood stabilizers, and/or } \\
\text { antipsychotics). UD relatives unmedicated }\end{array}$ & Color-word Stroop task & $\begin{array}{l}\text { Performance: no difference. Activity: BD (and relatives } \\
><\text { HC) } \downarrow \text { dIPFC connectivity with VACC. BD: positive } \\
\text { vIPFC-insula connectivity (negative connectivity in HC). } \downarrow \\
\text { vIPFC-basal ganglia connectivity in BD and UD relatives. }\end{array}$ \\
\hline Marchand et al $(2007)^{72}$ & $\begin{array}{l}\text { Case-control } \\
\text { study }\end{array}$ & $\begin{array}{l}14 \text { depressed BD } 1 \text { and } 15 \text { HC. BD medicated } \\
\text { (antidepressants, mood stabilizers, and/or } \\
\text { antipsychotics) }\end{array}$ & Color-word Stroop task & $\begin{array}{l}\text { Performance: no difference. Activity: BD } \downarrow \text { activity in } \\
\text { bilateral posterior cingulate and occipital cortex. }\end{array}$ \\
\hline Taylor et al (2016) $)^{100}$ & $\begin{array}{l}\text { Case-control } \\
\text { (3 sample } \\
\text { design) }\end{array}$ & $\begin{array}{l}16 \mathrm{UD} \text { in different phases and } 16 \mathrm{HC} \text { (and } 16 \mathrm{SZ} \text { ). } \\
63 \% \text { of UD medicated (antidepressants) }\end{array}$ & Color-word Stroop task & $\begin{array}{l}\text { Performance: UD } \downarrow \text { cognitive workload capacity }><H C \text {. } \\
\text { Activity: UD (and SZ) } \downarrow \text { functional connectivity } \\
\text { between ACC, parietal, and temporal hubs. }\end{array}$ \\
\hline Kikuchi et al $(2012)^{67}$ & $\begin{array}{l}\text { Case-control } \\
\text { study }\end{array}$ & 42 depressed UD and 17 HC. UD unmedicated & Color-word Stroop task & $\begin{array}{l}\text { Performance: no difference. Activity: UD: } \downarrow \text { activation } \\
\text { during incongruent blocks in middle frontal gyrus, } \\
\text { paracingulate and posterior cingulate, precuneus, } \\
\text { occipital regions, and brain stem. }\end{array}$ \\
\hline Wagner et al $(2010)^{106}$ & $\begin{array}{l}\text { Prospective, } \\
\text { naturalis- } \\
\text { tic open- } \\
\text { label } \\
\text { design }\end{array}$ & $\begin{array}{l}20 \text { depressed UD and } 20 \mathrm{HC} \text {. UD medicated } \\
\text { (benzodiazepines) }\end{array}$ & Color-word Stroop task & $\begin{array}{l}\text { Performance: no difference. Activity: UD } \boldsymbol{\uparrow} \text { froto-parieto- } \\
\text { temporal network and rostral ACC in the incongruent } \\
\text { condition. }\end{array}$ \\
\hline Wagner et al $(2006)^{107}$ & $\begin{array}{l}\text { Case-control } \\
\text { study }\end{array}$ & 16 depressed UD and 16 HC. UD unmedicated & Color-word Stroop task & $\begin{array}{l}\text { Performance: no difference. Activity: interference } \\
\text { condition: UD } \mathbb{T} \text { activity in rostral anterior cingulate } \\
\text { gyrus and left dIPFC; correlated with the Stroop } \\
\text { interference. Congruent condition: no difference. in } \\
\text { activity UD }><\text { HC }\end{array}$ \\
\hline Schlösser et al (2008) ${ }^{93}$ & $\begin{array}{l}\text { Case-control } \\
\text { study }\end{array}$ & 16 depressed UD and 16 HC. UD unmedicated & Color-word Stroop task & $\begin{array}{l}\text { Performance: no difference. Activity: UD } \boldsymbol{\uparrow} \text { dACC to } \mathrm{rACC} \\
\text { connectivity. UD: } \boldsymbol{\uparrow} \text { task-related input from the } \mathrm{dACC} \\
\text { to rACC. }\end{array}$ \\
\hline Strakowski et al $(2005)^{99}$ & $\begin{array}{l}\text { Case-control } \\
\text { study }\end{array}$ & $\begin{array}{l}16 \text { euthymic BD and } 16 \text { HC. BD medicated } \\
\text { (antidepressants, mood stabilizers, and/or } \\
\text { antipsychotics) }\end{array}$ & $\begin{array}{l}\text { Counting Stroop } \\
\text { interference task }\end{array}$ & $\begin{array}{l}\text { Performance: poorer in BD. Activity: BD: } \downarrow \text { activation in } \\
\text { temporal cortical regions (inferior, middle and } \\
\text { superior temporal gyrus), middle frontal gyrus, vIPFC, } \\
\text { putamen and midline cerebellum. BD } \mathbf{\text { medial }} \\
\text { occipital cortex. (HC: activation in right middle } \\
\text { temporal gurys = negatively correlated w. percentage } \\
\text { of correct responses and pos. corr. with false hits; BD: } \\
\text { reverse association.) }\end{array}$ \\
\hline Roth et al $(2006)^{91}$ & $\begin{array}{l}\text { Case-control } \\
\text { study }\end{array}$ & $\begin{array}{l}11 \text { mixed state, manic, depressed, or euthymic BD } \\
1 \text { and } 11 \text { HC. BD medicated (antidepressants, } \\
\text { mood stabilizers, and/or antipsychotics) }\end{array}$ & $\begin{array}{l}\text { Counting Stroop } \\
\text { interference task }\end{array}$ & $\begin{array}{l}\text { Performance: No difference. Activity: BD } \downarrow \text { activation in } \\
\text { right inferior gyrus, right pons, left posterior cingulate, } \\
\text { fusiform, parahippocampal, and middle occipital gyri. } \\
\text { BD } \downarrow \text { right posterior medial frontal gyrus. }\end{array}$ \\
\hline
\end{tabular}


TABLE 2. Continued

\begin{tabular}{|c|c|c|c|}
\hline Author & Study design & Participants & fMRI parad \\
\hline Penfold et al $(2015)^{82}$ & $\begin{array}{l}\text { Case-control } \\
\text { study }\end{array}$ & 19 depressed BD 2 and $20 \mathrm{HC}$. BD unmedicated & Go/no-go task \\
\hline Joshi et al (2016) & $\begin{array}{l}\text { Case-control } \\
\text { study }\end{array}$ & $\begin{array}{l}45 \text { euthymic BD } 1 \text { and } 45 \text { HC. BD medicated } \\
\text { (antidepressants, mood stabilizers, } \\
\text { antipsychotics, and/or benzodiazepines) }\end{array}$ & Go/no-go task \\
\hline Strakowski et al (2008) ${ }^{97}$ & $\begin{array}{l}\text { Case-control } \\
\text { study }\end{array}$ & $\begin{array}{l}19 \text { manic BD } 1 \text { and } 17 \text { HC. BD medicated } \\
\text { (antidepressants, mood stabilizers, and/or } \\
\text { antipsychotics) }\end{array}$ & Go/no-go task \\
\hline $\begin{array}{l}\text { Mazzola-Pomietto et al } \\
(2009)^{118}\end{array}$ & $\begin{array}{l}\text { Case-control } \\
\text { study }\end{array}$ & $\begin{array}{l}16 \text { manic BD and } 16 \mathrm{HC} \text {. } \\
\text { BD medicated (mood stabilizers and/or } \\
\text { antipsychotics) }\end{array}$ & Go/no-go task \\
\hline $\begin{array}{l}\text { Welander-Vatn et al } \\
\quad(2009)^{122}\end{array}$ & $\begin{array}{l}\text { Case-control } \\
\text { study }\end{array}$ & $\begin{array}{l}27 \text { euthymic, mildly or moderately/severely } \\
\text { depressed BD } 2 \text { and } 28 \text { HC. } 59 \% \text { of BD } \\
\text { medicated (antidepressants and/or mood } \\
\text { stabilizers) }\end{array}$ & Go/no-go task \\
\hline Townsend et al (2012) & $\begin{array}{l}\text { Case-control } \\
\text { study }\end{array}$ & $\begin{array}{l}32 \text { euthymic BD } 1 \text { and } 30 \mathrm{HC} .72 \% \text { of BD } \\
\text { medicated (antidepressants, mood stabilizers, } \\
\text { and/or antipsychotics) }\end{array}$ & Go/no-go task \\
\hline
\end{tabular}
and/or antipsychotics)

\begin{tabular}{|c|c|c|c|}
\hline Wessa et al (2007) ${ }^{112}$ & $\begin{array}{l}\text { Case-control } \\
\text { study }\end{array}$ & $\begin{array}{l}17 \text { euthymic BD and } 17 \mathrm{HC} . \mathrm{BD} \text { medicated } \\
\text { (antidepressants, mood stabilizers, and/or } \\
\text { antipsychotics) }\end{array}$ & Go/no-go task \\
\hline Ajilore et al $(2015)^{30}$ & $\begin{array}{l}\text { Case-control } \\
\text { study }\end{array}$ & $\begin{array}{l}16 \text { euthymic BD } 1 \text { and } 16 \text { HC. } 88 \% \text { of BD } \\
\text { medicated (antidepressants, mood stabilizers, } \\
\text { antipsychotics, and/or benzodiazepines) }\end{array}$ & Go/no-go task \\
\hline $\begin{array}{l}\text { Welander-Vatn et al } \\
\qquad(2013)^{110}\end{array}$ & $\begin{array}{l}\text { Case-control } \\
\text { study }\end{array}$ & $\begin{array}{l}24 \text { euthymic, depressed, or mixed manic/ } \\
\text { depressed BD } 1 \text { and } 24 \text { HC. BD medicated } \\
\text { (antidepressants, mood stabilizers, and/or } \\
\text { antipsychotics) }\end{array}$ & Go/no-go task \\
\hline Altshuler et al (2005) ${ }^{115}$ & $\begin{array}{l}\text { Case-control } \\
\text { study }\end{array}$ & $\begin{array}{l}11 \text { manic BD } 1 \text { and } 13 \mathrm{HC} \text {. BD medicated (mood } \\
\text { stabilizers and/or antipsychotics) }\end{array}$ & Go/no-go task \\
\hline Fleck et al (2011) & $\begin{array}{l}\text { Case-control } \\
\text { study (3- } \\
\text { sample } \\
\text { design) }\end{array}$ & $\begin{array}{l}20 \text { mixed episode or depressed BD } 1 \text { and } 10 \mathrm{HC} \text {. } \\
75 \% \text { of BD medicated (not specified) }\end{array}$ & Go/no-go task \\
\hline Kaladjian et al (2009) & $\begin{array}{l}\text { Case-control } \\
\text { study }\end{array}$ & $\begin{array}{l}20 \text { euthymic BD } 1 \text { and } 20 \mathrm{HC} \text {. BD medicated } \\
\text { (mood stabilizers and/or antipsychotics) }\end{array}$ & Go/no-go task \\
\hline $\begin{array}{l}\text { Korgaonkar et al } \\
\quad(2013)^{68}\end{array}$ & $\begin{array}{l}\text { Case-control } \\
\text { study }\end{array}$ & $\begin{array}{l}30 \text { depressed UD and } 30 \mathrm{HC} \text {. UD antidepressant } \\
\text { medication naîve }\end{array}$ & Go/no-go task \\
\hline Crane et al $(2016)^{40}$ & $\begin{array}{l}\text { Case-control } \\
\text { (3 sample } \\
\text { design) }\end{array}$ & $\begin{array}{l}47 \text { depressed UD ( } 29 \text { UD + anxiety) and } 54 \mathrm{HC} \text {. } \\
\text { UD unmedicated }\end{array}$ & Go/no-go task \\
\hline Rao et al $(2015)^{85}$ & $\begin{array}{l}\text { Case-control } \\
\text { study (4- } \\
\text { sample } \\
\text { design) }\end{array}$ & $\begin{array}{l}16 \text { depressed UD and } 18 \mathrm{HC} \text { (and } 20 \text { geriatric UD } \\
\text { and } 17 \text { geriatric } \mathrm{HC} \text { ). } 32 \% \text { of UD medicated } \\
\text { (not specified) }\end{array}$ & Go/no-go task \\
\hline Ryan et al (2015) ${ }^{92}$ & $\begin{array}{l}\text { Case-control } \\
\text { study }\end{array}$ & & Go/no-go task \\
\hline
\end{tabular}

Performance: no difference. Activity: NoGo $>$ Go contrast: $\mathrm{BD}(><\mathrm{HC}) \downarrow$ frontal activation in right inferior frontal gyrus, right middle frontal gyrus, superior frontal gyrus, insula, and bilateral precentral gyrus. $B D: \downarrow$ activation in the temporal and occipital lobes. Performance: no difference. Activity: During response inhibition, BD $\downarrow$ left prefrontal areas, right inferior parietal lobule, and left globus pallidus.

Performance: no difference. Activity: response inhibition: BD $\downarrow$ activation in anterior and posterior cingulate, medial dorsal thalamus, middle temporal gyrus, and precuneus.

Performance: poorer in BD in Go-trials. Activity: BD $\downarrow$ VIPFC during response inhibition.

Performance: no difference. Activity: no difference.

Performance: no difference. Activity: BD: $\downarrow$ activation in bilateral inferior frontal cortex, left medial frontal gyrus, left inferior and superior parietal lobe, bilateral putamen, bilateral caudate, bilateral globus pallidus, right thalamus, and right subthalamic nucleus. № areas of greater activation in $\mathrm{BD}$.

Performance: no diff. Activity: no diff.

Performance: poorer in BD. Activity: BD $\downarrow$ vIPFC activation during No-Go minus Go, driven primarily by the right side.

Performance: poorer in BD. Activity: no difference. In either full brain analysis or region of interest approach.

Performance: no difference. Activity: BD $\downarrow$ activation in right lateral orbitofrontal cortex, right IPFC, and right hippocampus. BD $\downarrow$ activation in left rostral cingulate.

Performance: Poorer in BD. Activity: BD-Mixed Tactivation of right amygdala and frontal cortex ( $><\mathrm{HC}$ ). BD-mixed $\mathbf{\uparrow}$ left thalamus, left cerebellum, and right inferior frontal gyrus ( $><$ BD-Depressed).

Performance: no difference. Activity: BD $\downarrow$ activation in left frontopolar cortex and bilateral dorsal amygdala during response inhibition.

Performance: poorer in UD. Activity: UD $\uparrow$ ACC during response inhibition.

Performance: poorer in UD on Go-trials. Activity: UD $\downarrow$ right inferior frontal gyrus during commissions and $\downarrow$ throughout the brain (superior and middle frontal regions, posterior cingulate, cuneus, fusiform, and caudate) during rejection.

Performance: poorer in UD. Activity (during correct hits): UD $\mathbb{T}$ activation in the left cuneus $><$ HC.

Performance: poorer in BD and UD (BD worse). Activity: $\mathrm{BD}$ and UD: $\mathbf{\uparrow}$ activation in left superior temporal 
TABLE 2. Continued

\begin{tabular}{|c|c|c|c|}
\hline Author & Study design & Participants & fMRI paradigm \\
\hline & & $\begin{array}{l}19 \text { depressed or euthymic UD, } 16 \text { depressed or } \\
\text { euthymic BD, and } 17 \mathrm{HC} \text {. Medication not } \\
\text { specified }\end{array}$ & \\
\hline Mclntosh et al (2008) & $\begin{array}{c}\text { Case-control } \\
\text { study (3- } \\
\text { sample } \\
\text { design) }\end{array}$ & $\begin{array}{l}42 \text { euthymic BD } 1 \text { and } 37 \mathrm{HC} \text { (and } 27 \text { SZ). BD } \\
\text { medicated (antidepressants, mood stabilizers, } \\
\text { and/or antipsychotics) }\end{array}$ & $\begin{array}{l}\text { Hayling Sentence } \\
\text { Completion Test }\end{array}$ \\
\hline
\end{tabular}

\begin{tabular}{|c|c|c|c|}
\hline Gruber et al $(2017)^{56}$ & $\begin{array}{l}\text { Case-control } \\
\text { study }\end{array}$ & $\begin{array}{l}29 \text { euthymic BD (some with residual depressive } \\
\text { symptoms) and } 21 \text { HC. BD medicated } \\
\text { (antidepressants, mood stabilizers, } \\
\text { antipsychotics, and/or benzodiazepines) }\end{array}$ & $\begin{array}{l}\text { Multi-source } \\
\text { interference task }\end{array}$ \\
\hline Weathers et al (2013) ${ }^{109}$ & $\begin{array}{l}\text { Case-control } \\
\text { study (4- } \\
\text { sample } \\
\text { design) }\end{array}$ & $\begin{array}{l}23 \text { euthymic, depressed, hypomanic, or mixed } \\
\text { state BD and } 27 \mathrm{HC} \text { (and } 15 \text { pediatric } \mathrm{BD} \text { and } \\
20 \text { pediatric } \mathrm{HC} \text { ). BD medicated } \\
\text { (antidepressants, mood stabilizers, } \\
\text { antipsychotics, and/or benzodiazepines) }\end{array}$ & $\begin{array}{l}\text { Response flexibility } \\
\text { paradigm }\end{array}$ \\
\hline Remijnse et al (2013) ${ }^{86}$ & $\begin{array}{l}\text { Case-control } \\
\text { study }\end{array}$ & $\begin{array}{l}19 \text { depressed UD and } 29 \mathrm{HC} \text { (and } 18 \text { OCD). UD } \\
\text { unmedicated }\end{array}$ & $\begin{array}{l}\text { Self-paced letter/digit } \\
\text { task switching } \\
\text { paradigm }\end{array}$ \\
\hline van Tol et al (2011) $)^{104}$ & $\begin{array}{l}\text { Case-control } \\
\text { study }\end{array}$ & $\begin{array}{l}65 \text { remitted, mildly depressed, or moderately/ } \\
\text { severely depressed UD, } 82 \text { UD + anxiety, and } \\
64 \text { HC (and } 64 \text { anxiety). } 25 \% \text { of UD medicated } \\
\text { (antidepressants) }\end{array}$ & Tower of London task \\
\hline Fitzgerald et al $(2008)^{48}$ & $\begin{array}{l}\text { Case-control } \\
\text { study }\end{array}$ & $\begin{array}{l}13 \text { depressed UD and } 13 \text { HC. 85\% of UD } \\
\text { medicated (antidepressants) }\end{array}$ & Tower of London task \\
\hline Rive et al $(2016)^{87}$ & $\begin{array}{l}\text { Case-control } \\
\text { study (5- } \\
\text { sample } \\
\text { design) }\end{array}$ & $\begin{array}{l}40 \text { remitted or depressed UD, } 32 \text { remitted or } \\
\text { depressed } \mathrm{BD} \text {, and } 35 \mathrm{HC} \text {. UD and BD } \\
\text { unmedicated }\end{array}$ & Tower of London task \\
\hline
\end{tabular}

\begin{tabular}{|c|c|c|c|c|}
\hline Curtis et al (2007) & $\begin{array}{l}\text { Case-control } \\
\text { study }\end{array}$ & $\begin{array}{l}12 \text { euthymic BD and } 12 \text { HC. BD medicated (mood } \\
\text { stabilizers) }\end{array}$ & $\begin{array}{l}\text { Phonetic lexical decision } \\
\text { task: rhyming task, } \\
\text { semantic lexical } \\
\text { decision task, } \\
\text { phonetic verbal } \\
\text { fluency task, } \\
\text { semantic verbal } \\
\text { fluency task }\end{array}$ & $\begin{array}{l}\text { and depressed UD (hypo-activation }><\mathrm{HC} \text { )]. } \\
\text { Performance: poorer in BD. Activity: BD showed } \uparrow \\
\text { activation in left PFC and bilateral cerebellum/ } \\
\text { fusiform/lingual gyrus cluster (medial occipital } \\
\text { cortex). BD: } \downarrow \text { activity in medial frontal cortex incl. } \\
\text { ACC. BD } \uparrow \text { PFC response to high-demand tasks } \\
\text { compared to low-demand tasks ( }><\mathrm{HC} \text { ). }\end{array}$ \\
\hline $\begin{array}{l}\text { Yoshimura et al } \\
\qquad(2014)^{114}\end{array}$ & $\begin{array}{l}\text { Case-control } \\
\text { study }\end{array}$ & $\begin{array}{l}10 \text { euthymic BD } 1 \text { and } 10 \mathrm{HC} . \mathrm{BD} \text { medicated } \\
\text { (mood stabilizers and/or antipsychotics) }\end{array}$ & Verbal fluency task & $\begin{array}{l}\text { Performance: no difference. Activity: BD } \uparrow \text { activation in } \\
\text { the bilateral precuneus. }\end{array}$ \\
\hline Allin et al $(2010)^{31}$ & $\begin{array}{l}\text { Case-control } \\
\text { study }\end{array}$ & $\begin{array}{l}18 \text { remitted } \mathrm{BD}, 19 \text { relatives, and } 19 \mathrm{HC} .74 \% \text { of } \\
\text { BD medicated (antidepressants, mood } \\
\text { stabilizers, and/or antipsychotics) }\end{array}$ & Verbal fluency task & $\begin{array}{l}\text { Performance: poorer in BD. Activity: BD } \uparrow \text { activation in } \\
\text { the posterior cingulate cortex and } \downarrow \text { left PFC activity } \\
(><\mathrm{HC}) \text {. }\end{array}$ \\
\hline $\begin{array}{l}\text { Costafreda et al } \\
\qquad(2011)^{39}\end{array}$ & $\begin{array}{l}\text { Case-control } \\
\text { study }\end{array}$ & $\begin{array}{l}32 \text { euthymic BD and } 40 \mathrm{HC} \text { (and } 32 \mathrm{SZ} \text { ). } 81 \% \text { of } \\
\text { BD medicated (antidepressants, mood } \\
\text { stabilizers, and/or antipsychotics) }\end{array}$ & Verbal fluency task & $\begin{array}{l}\text { Performance: no difference. Activity: BD } \uparrow \text { activation in } \\
\text { dorsal anterior cingulate, dIPFC, and right putamen } \\
\text { (intermediate that of HC and SZ). BD } \uparrow \text { activity in the } \\
\text { precuneus, posterior cingulate, and angular gyrus ( } \downarrow \\
\text { deactivation). }\end{array}$ \\
\hline
\end{tabular}


TABLE 2. Continued

\begin{tabular}{|c|c|c|c|c|}
\hline Author & Study design & Participants & fMRI paradigm & Findings \\
\hline Okada et al (2003) ${ }^{120}$ & $\begin{array}{l}\text { Case-control } \\
\text { study }\end{array}$ & $\begin{array}{l}10 \text { depressed UD and } 10 \text { HC. UD medicated } \\
\text { (antidepressants) }\end{array}$ & Verbal fluency task & $\begin{array}{l}\text { Performance: poorer in UD. Activity: UD } \downarrow \text { activation of } \\
\text { ACC and inferior frontal gyrus. }\end{array}$ \\
\hline Hugdahl et al (2004) ${ }^{61}$ & $\begin{array}{l}\text { Case-control } \\
\text { study }\end{array}$ & $\begin{array}{l}12 \text { depressed UD and } 12 \text { HC (and } 12 \text { SZ). UD } \\
\text { medicated (antidepressants) }\end{array}$ & $\begin{array}{l}\text { Vigilance task and a } \\
\text { mental arithmetic } \\
\text { task }\end{array}$ & $\begin{array}{l}\text { Performance: poorer in UD in both tasks. Activity: UD } \uparrow \\
\text { activity in middle frontal gyrus. UD: } \downarrow \text { activation in the } \\
\text { right inferior parietal lobule. }\end{array}$ \\
\hline
\end{tabular}

Abbreviations: HC: Healthy control, BD: Bipolar disorder, UD: Unipolar disorder, SZ: Schizophrenia, OCD: Obsessive compulsive disorder, PFC: prefrontal cortex, dIPFC: dorsolateral PFC, vIPFC: ventrolateral PFC, ACC: Anterior cingulate cortex, vACC: Ventral ACC, rACC: rostral ACC, Anterior cingulate gyrus, IPG: inferior parietal gyrus, SPG: superior parietal gyrus, sig. diff: Significant difference, $><$ : Compared to.

Working memory in unipolar disorder. Six $(43 \%)$ studies found working memory impairments in symptomatic and remitted UD, ${ }^{52,59,71,89,105,108}$ while 8 (57\%) showed comparable performance in UD and HC. ${ }^{34,35,48,60,73,78,90,94}$ As with BD, the most consistent neural activation differences during working memory were altered response of cognitive control areas, most consistently in the dIPFC $^{48,52,60,73,89,108}$ and impaired deactivation of DMN regions, including the medial PFC. ${ }^{35,89,90}$

Of 6 studies in symptomatic and partially remitted patients, 2 studies found dlPFG hypo-activation, which was associated with impaired performance, ${ }^{52,89}$ while another 4 found dIPFC hyper-activity that was accompanied by preserved performance. ${ }^{48,60,73,108}$ However, this association between direction of dlPFC activity and performance was not uniform; 3 studies reported no increase in dlPFC in remitted patients with intact working memory performance, ${ }^{34,78,94}$ and 1 study found no dIPFC hypo-activation in cognitively impaired patients. ${ }^{59}$ Notably, 2 of the 3 studies showing intact working memory performance and normal dlPFC activity were conducted in remitted patients, suggesting that cognitive and neural functioning is normalized after remission in some UD patients ${ }^{34,94}$. Finally, symptomatic and remitted UD patients were found to display altered FG within PFC regions and between PFC and parietal nodes of the cognitive control network. ${ }^{35,71,105}$

\section{Executive skills}

Forty-three studies examined executive skills using a variety of different tasks, most commonly the Stroop, Go/No-Go, Tower of London, and Verbal fluency tasks. Twenty-eight studies were conducted in patients with BD and 13 in patients with UD, while 2 studies examined both populations (see Table 2).

Executive skills in bipolar disorder. Seventeen (61\%) studies in BD reported no behavioral differences from
HC, while 11 (39\%) studies demonstrated poorer performance in BD (see Table 2). Of these, most studies that reported no behavioral differences were conducted in remitted patients.

A highly consistent finding across $75 \%$ of the studies was hypo-activity within a distributed cognitive control network including vlPFC/inferior frontal gyrus, dlPFC, and inferior and superior parietal areas, which appeared to be largely independent of mood state and performance levels, ${ }^{30,31,36,39,50,64,69,82,84,91,92,99,109,115,117,118,121}$

with only $25 \%$ of studies reporting hyper-activation in these regions. ${ }^{41,56,57,87,116,119}$ Hypo-activation was particularly pronounced in prefrontal, parietal, and striatal regions. ${ }^{30,31,36,50,64,69,82,84,91,99,109,115,117,118,121}$ The findings indicate that hypo-activation in the cognitive control network in BD can occur even when the task load does not exceed patients' cognitive capacity.

Abnormal task-related anterior cingulate cortex (ACC) activity, particularly in the dorsal part, was also reported in multiple BD studies across mood states. Again, the majority of these studies reported hypo-activation, ${ }^{41,56,57,92,97,115}$ while 2 reported hyper-activation ${ }^{39}$ or no differences. ${ }^{110} \mathrm{~A}$ final replicated finding was decreased task-related ventral ACG-PFG FC in remitted BD. ${ }^{50,83}$

Executive skills in unipolar disorder. Nine (69\%) studies of UD found impaired task performance, ${ }^{40,48,61,68,85,86,100,104,120}$ particularly in patients with greater depression severity, $^{104}$ while $4(31 \%)$ studies ${ }^{67,93,106,107}$ reported no behavioral differences. These studies were almost all conducted in symptomatic patients. In general, patients exhibited hypo-activation in prefrontal and parietal cognitive control regions when performance was impaired and hyper-activation in this network when performance was preserved, ${ }^{40,86,87,104,106,107,120}$ although 2 studies found hyper-activity in these regions in patients with impaired performance. ${ }^{48,92}$ The areas with most consistent activation abnormalities were inferior, middle, and frontal gyrus; anterior PFC; dlPFC; and inferior parietal cortex. In addition, symptomatic patients also 
TABLE 3. Summary of included studies for the cognitive domains: learning and memory, attention, and implicit learning

\begin{tabular}{|c|c|c|c|c|}
\hline Author & Study design & Participants & fMRI paradigm & Findings \\
\hline \multicolumn{5}{|l|}{ Learning and memory } \\
\hline Glahn et al $(2010)^{54}$ & $\begin{array}{l}\text { Case-control } \\
\text { study }\end{array}$ & $\begin{array}{l}15 \text { remitted BD } 1 \text { and } 24 \text { HC. } 93 \% \text { of BD medicated } \\
\text { (antidepressants, mood stabilizers, and/or } \\
\text { antipsychotics) }\end{array}$ & $\begin{array}{l}\text { Associative learning } \\
\text { paradigm }\end{array}$ & $\begin{array}{l}\text { Performance: no difference. Activity: BD: } \downarrow \text { encoding- } \\
\text { related activation in left inferior frontal gyrus, } \\
\text { cingulate gyrus, superior parietal lobule, right } \\
\text { insular cortex, lentiform nucleus, and bilateral } \\
\text { occipital and cerebellum and } \ \text { activation in left } \\
\text { middle frontal gyrus, precuneus, and left superior } \\
\text { temporal gyrus. BD } \downarrow \text { retrieval-related left } \\
\text { hippocampus and parahippocampal gyrus, } \\
\text { bilateral cerebellum, bilateral sensory-motor } \\
\text { regions. Region of interest analyses: BD } \uparrow \\
\text { encoding-related and } \downarrow \text { retrieval-related activity in } \\
\text { dIPFC. }\end{array}$ \\
\hline Hall et al $(2010)^{58}$ & $\begin{array}{l}\text { Case-control } \\
\text { study }\end{array}$ & $\begin{array}{l}14 \text { stable BD } 1 \text { and } 14 \mathrm{HC} \text { (and } 15 \mathrm{SZ} \text { ). BD medicated } \\
\text { (antidepressants, mood stabilizers, and/or } \\
\text { antipsychotics) }\end{array}$ & $\begin{array}{l}\text { Associative learning } \\
\text { paradigm }\end{array}$ & $\begin{array}{l}\text { Performance: no difference (was matched). Activity: } \\
\text { BD } \downarrow \text { activation in left dIPFC during encoding }>< \\
\text { HC (and SZ). }\end{array}$ \\
\hline Werner et al (2009) ${ }^{111}$ & $\begin{array}{l}\text { Case-control } \\
\text { study }\end{array}$ & $\begin{array}{l}11 \text { depressed UD and } 11 \text { HC. UD medicated } \\
\text { (antidepressants, mood stabilizers, and/or } \\
\text { antipsychotics) }\end{array}$ & $\begin{array}{l}\text { Associative learning } \\
\text { paradigm }\end{array}$ & $\begin{array}{l}\text { Performance: no difference. Activity: UD } \boldsymbol{\uparrow} \text { encoding- } \\
\text { related activity in superior part of left } \\
\text { parahippocampal gyrus and } \downarrow \text { activity in frontal } \\
\text { and parietal regions. UD } \downarrow \text { retrieval-related } \\
\text { activation in ACC and parietal areas and } \boldsymbol{\uparrow} \text { activity } \\
\text { in left superior frontal gyrus and right fusiform } \\
\text { gyrus. }\end{array}$ \\
\hline Fairhall et al $(2010)^{46}$ & $\begin{array}{l}\text { Case-control } \\
\text { study }\end{array}$ & $\begin{array}{l}8 \text { depressed UD and } 8 \text { HC. } 75 \% \text { of UD medicated } \\
\text { (antidepressants and/or benzodiazepines) }\end{array}$ & $\begin{array}{l}\text { Associative learning } \\
\text { paradigm }\end{array}$ & $\begin{array}{l}\text { Performance: no difference. Activity: significant group } \\
\text { × condition interaction in bilateral anterior } \\
\text { hippocampus: positive relationship between } \\
\text { hippocampal activity and successful encoding in } \\
\text { HC; not present in UD. UD: dysregulated memory } \\
\text { related hippocampal function rather than hypo- or } \\
\text { hyper-activation of hippocampus per se. }\end{array}$ \\
\hline $\begin{array}{l}\text { Oertel-Knöchel et al } \\
\qquad(2014)^{80}\end{array}$ & $\begin{array}{l}\text { Case-control } \\
\text { study }\end{array}$ & $\begin{array}{l}21 \text { remitted BD } 1 \text { and } 20 \mathrm{HC} \text {. BD medicated } \\
\text { (antidepressants, mood stabilizers, } \\
\text { antipsychotics, and/or benzodiazepines) }\end{array}$ & $\begin{array}{l}\text { Computer-based } \\
\text { nonverbal learning } \\
\text { and recognition } \\
\text { test }\end{array}$ & $\begin{array}{l}\text { Performance: poorer in BD. Activity: BD } \downarrow \text { encoding- } \\
\text { related activity in bilateral ACC, precuneus/ } \\
\text { cuneus, and left lingual gyrus. BD } \mathbf{t} \text { activation ( } \downarrow \\
\text { deactivation) in left temporo-parietal junction and } \\
\downarrow \downarrow \text { ventral hippocampal activation during retrieval. }\end{array}$ \\
\hline Dietsche et al (2014) & $\begin{array}{l}\text { Case-control } \\
\text { study }\end{array}$ & $\begin{array}{l}23 \text { depressed UD and } 23 \text { HC. UD medicated } \\
\text { (antidepressants and/or antipsychotics) }\end{array}$ & $\begin{array}{l}\text { Non-emotional } \\
\text { episodic memory } \\
\text { encoding and } \\
\text { retrieval task }\end{array}$ & $\begin{array}{l}\text { Performance: poorer in UD. Activity: UD } \downarrow \text { encoding- } \\
\text { related activation in right middle and medial } \\
\text { frontal gyrus, right cingulate cortex, hippocampus, } \\
\text { and parahippocampal gyrus. UD } \mathbf{t} \text { retrieval-related } \\
\text { activation in the right inferior frontal gyrus. Lack of } \\
\text { "normal" association between encoding-related } \\
\text { hippocampal activation and retrieval success in } \\
\text { UD. }\end{array}$ \\
\hline Kassel et al (2016) ${ }^{65}$ & $\begin{array}{l}\text { Case-control } \\
\text { study }\end{array}$ & $\begin{array}{l}42 \text { UD in different phases and } 40 \mathrm{HC} \text {. } 41 \% \text { of UD } \\
\text { medicated (not specified) }\end{array}$ & $\begin{array}{l}\text { Semantic list learning } \\
\text { task }\end{array}$ & $\begin{array}{l}\text { Performance: poorer in UD. Activity: UD } \downarrow \text { encoding- } \\
\text { specific activation in bilateral middle frontal gyrus, } \\
\text { dorsal ACC, insula, precuneus, superior parietal } \\
\text { lobule, thalamus, and cerebellum. }\end{array}$ \\
\hline $\begin{array}{l}\text { van Eijndhoven et al } \\
\quad(2013)^{102}\end{array}$ & $\begin{array}{l}\text { Case-control } \\
\text { study }\end{array}$ & $\begin{array}{l}40 \text { recovered or depressed medication free UD and } 20 \\
\text { HC }\end{array}$ & $\begin{array}{l}\text { Source recollection } \\
\text { paradigm and } \\
\text { Picture encoding } \\
\text { and recognition } \\
\text { task }\end{array}$ & $\begin{array}{l}\text { Performance: no difference. Activity: symptomatic UD } \\
\mathbf{T} \text { retrieval-related activity in left inferior frontal } \\
\text { gyrus. }\end{array}$ \\
\hline $\begin{array}{l}\text { van Eijndhoven et al } \\
\qquad(2011)^{103}\end{array}$ & $\begin{array}{l}\text { Case-control } \\
\text { study }\end{array}$ & $\begin{array}{l}40 \text { recovered or depressed UD and } 20 \mathrm{HC} \text {. Depressed } \\
\text { subgroup: antidepressant medication naïve and } \\
\text { recovered subgroup medication-free }\end{array}$ & $\begin{array}{l}\text { Source recollection } \\
\text { paradigm and } \\
\text { Picture encoding } \\
\text { and recognition } \\
\text { task }\end{array}$ & $\begin{array}{l}\text { Performance: no difference. Activity: symptomatic } \\
\text { patients } \uparrow \text { activation in left inferior frontal gyrus, } \\
\text { left insula, posterior cingulate, precuneus, caudate } \\
\text { nucleus, and bilateral thalamus }><\text { HC and } \\
\text { remitted patients. Greater memory-related right } \\
\text { amygdala activity in all patients ( }><\mathrm{HC} \text { ). }\end{array}$ \\
\hline $\begin{array}{l}\text { Finkelmeyer et al } \\
\qquad(2016)^{47}\end{array}$ & $\begin{array}{l}\text { Case-control } \\
\text { study }\end{array}$ & $\begin{array}{l}20 \text { depressed UD and } 20 \text { HC. UD medicated } \\
\text { (antidepressants, mood stabilizers, } \\
\text { antipsychotics, and/or benzodiazepines) }\end{array}$ & Spatial memory task & $\begin{array}{l}\text { Performance: no difference. Activity: UD failed to show } \\
\text { normal task-dependent changes bilateral anterior } \\
\text { hippocampus activity but no activity differences in } \\
\text { the whole-brain analyses. }\end{array}$ \\
\hline
\end{tabular}


TABLE 3. Continued

\begin{tabular}{|c|c|c|c|c|}
\hline Author & Study design & Participants & fMRI paradigm & Findings \\
\hline Milne et al $(2012)^{76}$ & $\begin{array}{l}\text { Case-control } \\
\text { study }\end{array}$ & $\begin{array}{l}22 \text { euthymic UD and } 18 \text { HC. } 86 \% \text { of UD medicated } \\
\text { (antidepressants, mood stabilizers, and/or } \\
\text { antipsychotics) }\end{array}$ & $\begin{array}{l}\text { Recollection memory } \\
\text { process } \\
\text { dissociation task }\end{array}$ & $\begin{array}{l}\text { Performance: poorer in UD. Activity: UD } \downarrow \text { activation of } \\
\text { the right hippocampal and left parahippocampal } \\
\text { gyrus during recollection. (Recollection memory } \\
\text { performance was corr. with changes in right } \\
\text { hippocampus BOLD signal in HC; but not UD.) }\end{array}$ \\
\hline Meusel et al $(2013)^{24}$ & $\begin{array}{l}\text { Case-control } \\
\text { study and } \\
\text { non- } \\
\text { randomized } \\
\text { clinical trial }\end{array}$ & $\begin{array}{l}38 \text { partially remitted BD/UD and } 18 \text { HC. BD/UD } \\
\text { medicated (antidepressants, mood stabilizers, } \\
\text { antipsychotics, and/or benzodiazepines) }\end{array}$ & $\begin{array}{l}\text { Recollection memory } \\
\text { process } \\
\text { dissociation task }\end{array}$ & $\begin{array}{l}\text { Performance: poorer in BD/UD. Activity: BD/UD } \downarrow \\
\text { recollection-specific activation in anterior } \\
\text { parahippocampal gyrus. }\end{array}$ \\
\hline Jamadar et al $(2013)^{62}$ & $\begin{array}{l}\text { Case-control } \\
\text { study }\end{array}$ & $\begin{array}{l}32 \text { BD (phase not specified) and } 133 \mathrm{HC} \text { (and } 74 \mathrm{SZ} \text { ). } \\
\text { BD medicated (antidepressants, mood } \\
\text { stabilizers, antipsychotics, and/or } \\
\text { benzodiazepines) }\end{array}$ & $\begin{array}{r}\text { Semantic object } \\
\text { retrieval task }\end{array}$ & $\begin{array}{l}\text { Performance: poorer in BD. Activity: BD } \downarrow \text { retrieval- } \\
\text { specific inferior parietal lobule. }\end{array}$ \\
\hline Kelley et al (2013) ${ }^{66}$ & $\begin{array}{l}\text { Case-control } \\
\text { study }\end{array}$ & $\begin{array}{l}16 \text { depressed, psychotic UD, } 15 \text { depressed, } \\
\text { nonpsychotic UD, and } 16 \text { HC. 68\% of UD } \\
\text { medicated (antidepressants, mood stabilizers, } \\
\text { antipsychotics and/or benzodiazepines) }\end{array}$ & $\begin{array}{l}\text { Verbal declarative } \\
\text { memory task }\end{array}$ & $\begin{array}{l}\text { Performance: only poorer in psychotic UD ( }><\mathrm{HC} \text { and } \\
\text { non-psychotic UD). Activity: all UD } \downarrow \text { right ACC } \\
\text { activity during encoding and retrieval. Psychotic } \\
\text { UD } \downarrow \text { encoding-related hippocampus, insula, and } \\
\text { middle frontal gyrus activity and } \uparrow \text { retrieval- } \\
\text { related PFC and parietal activation }><\mathrm{HC} \text { and } \\
\text { nonpsychotic UD. }\end{array}$ \\
\hline $\begin{array}{l}\text { Oertel-Knöchel et al } \\
\qquad(2013)^{79}\end{array}$ & $\begin{array}{l}\text { Case-control } \\
\text { study }\end{array}$ & $\begin{array}{l}26 \text { remitted BD } 1 \text { and } 25 \mathrm{HC} . \mathrm{BD} \text { medicated } \\
\text { (antidepressants, mood stabilizers, } \\
\text { antipsychotics, and/or benzodiazepines) }\end{array}$ & $\begin{array}{l}\text { Verbal learning and } \\
\text { recognition test }\end{array}$ & $\begin{array}{l}\text { Performance: poorer in BD. Activity: BD } \downarrow \text { encoding- } \\
\text { related activity in left middle and superior frontal } \\
\text { gyrus. BD } \downarrow \text { retrieval-related activity in middle and } \\
\text { inferior frontal gyrus, precuneus, cuneus, } \\
\text { parahippocampal gyrus and the posterior } \\
\text { cingulate, and in the caudate nucleus. }\end{array}$ \\
\hline \multicolumn{5}{|l|}{ Attention } \\
\hline Smucny et al (2017) & $\begin{array}{l}\text { Case-control (3 } \\
\text { sample } \\
\text { design) }\end{array}$ & $\begin{array}{l}24 \text { BD } 1 \text { with psychotic features (phase not } \\
\text { specified) and } 53 \mathrm{HC} \text { (and } 70 \text { SZ). BD medicated } \\
\text { (mood stabilizers and/or antipsychotics) }\end{array}$ & $\begin{array}{l}\text { AX-continuous } \\
\text { performance task }\end{array}$ & $\begin{array}{l}\text { Performance: trend-level impairment in BD. Activity: } \\
\text { combined BD and SZ group } \downarrow \text { dIPFC and superior } \\
\text { parietal response (no difference between } S Z \text { and } \\
\text { BD) and BD also } \downarrow \text { ACC activity ( }><H C \text { ). }\end{array}$ \\
\hline Fleck et al $(2012)^{49}$ & $\begin{array}{l}\text { Case-control } \\
\text { study }\end{array}$ & $\begin{array}{l}50 \text { manic/mixed BD } 1 \text { and } 34 \text { HC. BD medicated } \\
\text { (antidepressants, mood stabilizers, } \\
\text { antipsychotics, and/or benzodiazepines) }\end{array}$ & $\begin{array}{l}\text { Continuous } \\
\text { performance task }\end{array}$ & $\begin{array}{l}\text { Performance: BD: poorer sustained performance. } \\
\text { Activity: BD } \uparrow \text { bilateral amygdala activation and } \downarrow \\
\text { activity over time in dorsal and ventral regions of } \\
\text { an anterior-limbic network and in left striatum } \\
\text { thalamus. }\end{array}$ \\
\hline Strakowski et al (2004) ${ }^{98}$ & $\begin{array}{l}\text { Case-control } \\
\text { study }\end{array}$ & 10 euthymic unmedicated BD and $10 \mathrm{HC}$. & $\begin{array}{l}\text { Continuous } \\
\text { performance task }\end{array}$ & $\begin{array}{l}\text { Performance: no difference. Activity: BD } \downarrow \text { activity in } \\
\text { medial frontal cortex and fusiform gyrus and } \uparrow \\
\text { inferior frontal cortex and vIPFC regions and limbic } \\
\text { regions, insula, postcentral, occipito-temporal, and } \\
\text { parietal cortex. }\end{array}$ \\
\hline $\begin{array}{l}\text { Korgaonkar et al } \\
\qquad(2013)^{68}\end{array}$ & $\begin{array}{l}\text { Case-control } \\
\text { study }\end{array}$ & $\begin{array}{l}30 \text { depressed UD and } 30 \text { HC. UD antidepressant- } \\
\text { naïve. }\end{array}$ & $\begin{array}{l}\text { Continuous } \\
\text { performance task }\end{array}$ & $\begin{array}{l}\text { Performance: poorer in UD. Activity: UD } \uparrow \text { ACC activity } \\
\text { and } \downarrow \text { right dIPFC activity. }\end{array}$ \\
\hline Desseilles et al $(2011)^{44}$ & $\begin{array}{l}\text { Case-control } \\
\text { study }\end{array}$ & 14 depressed UD and 14 HC. UD unmedicated & Detection task & $\begin{array}{l}\text { Performance: no difference. Activity: Aberrant effective } \\
\text { connectivity, with decreased parietal top-down } \\
\text { modulation of early occipital processing of visual } \\
\text { stimuli. }\end{array}$ \\
\hline Desseilles et al (2009) & $\begin{array}{l}\text { Case-control } \\
\text { study }\end{array}$ & 14 depressed UD and 14 HC. UD unmedicated & Detection task & $\begin{array}{l}\text { Performance: poorer in UD in high-load conditions. } \\
\text { Activity: UD } ₫ \text { subgenual cingulate/medial } \\
\text { orbitofrontal cortex activity and } \downarrow \text { functional } \\
\text { connectivity between fronto-parietal networks and } \\
\text { visual cortices. UD: lack of load-related increased } \\
\text { coupling between parietal or frontal regions and } \\
\text { visual cortices. }\end{array}$ \\
\hline $\begin{array}{l}\text { Korgaonkar et al } \\
\qquad(2013)^{68} \\
\text { Implicit learning }\end{array}$ & $\begin{array}{l}\text { Case-control } \\
\text { study }\end{array}$ & $\begin{array}{l}30 \text { depressed UD and } 30 \text { HC. UD antidepressant- } \\
\text { naïve. }\end{array}$ & Oddball task & $\begin{array}{l}\text { Performance: poorer in UD. Activity: No difference in } \\
\text { prefrontal activity. }\end{array}$ \\
\hline Naismith et al $(2010)^{77}$ & $\begin{array}{l}\text { Case-control } \\
\text { study }\end{array}$ & $\begin{array}{l}19 \text { depressed UD and } 20 \text { HC. } 89 \% \text { of UD medicated } \\
\text { (antidepressants, mood stabilizers, } \\
\text { antipsychotics, and/or benzodiazepines) }\end{array}$ & $\begin{array}{l}\text { Motor sequencing } \\
\text { implicit learning } \\
\text { task }\end{array}$ & $\begin{array}{l}\text { Performance: UD impaired implicit learning. Activity: } \\
\text { UD } \downarrow \text { dIPFC and } \mathbf{\uparrow} \text { superior temporal gyrus and } \\
\text { cerebellum activity. }\end{array}$ \\
\hline
\end{tabular}


TABLE 3. Continued

\begin{tabular}{|c|c|c|c|c|}
\hline Author & Study design & Participants & fMRI paradigm & Findings \\
\hline Genzel et al $(2015)^{53}$ & $\begin{array}{l}\text { Case-control (3 } \\
\text { sample } \\
\text { design) }\end{array}$ & $\begin{array}{l}16 \text { depressed UD and } 16 \mathrm{HC} \text { (and } 16 \mathrm{SZ} \text { ). UD } \\
\text { medicated (antidepressants, antipsychotics, and/ } \\
\text { or benzodiazepines) }\end{array}$ & $\begin{array}{l}\text { Sequential finger- } \\
\text { tapping task }\end{array}$ & $\begin{array}{l}\text { Performance: UD impaired implicit learning. Activity: } \\
\text { UD } \downarrow \text { task-induced deactivation of DMN and } \downarrow \\
\text { hippocampus-PFC connectivity during the task } \\
\text { performance. After one night of memory } \\
\text { consolidation: UD failed to show normal overnight } \\
\downarrow \text { activation of basal ganglia and PFC. }\end{array}$ \\
\hline
\end{tabular}

Abbreviations: HC: Healthy control, BD: Bipolar disorder, UD: Unipolar disorder, SZ: Schizophrenia, PFC: prefrontal cortex, dIPFC: dorsolateral PFC, vIPFC: ventrolateral PFC, ACC: Anterior cingulate cortex, sig. diff: Significant difference, $><$ : Compared to.

displayed hyper-activity in dorsal and rostral ACC activation, ${ }^{68,92,106,107}$ although 1 study observed ACC hypoactivation. ${ }^{120}$ As in BD, UD patients also showed altered FC within the ACG and between ACC and parietal and temporal hubs. ${ }^{93,100}$ Finally, a study investigating both UD and BD in depressed or remitted states in comparison with $\mathrm{HC}$ reported more pronounced executive deficits in BD than in UD, which were coupled with hypo-activity in $\mathrm{BD}$ and hyper-activity in UD. ${ }^{92}$

\section{Learning and memory}

Fifteen studies investigated learning and memory: 5 in $\mathrm{BD},{ }^{54,58,62,79,80} 9$ in UD, ${ }^{13,46,47,65,66,76,102,103,111}$ and 1 in both $^{24}$ (see Table 3 ).

Learning and memory in bipolar disorder. Three (60\%) studies of remitted BD patients found impaired performance across verbal and nonverbal memory tasks, ${ }^{62,79,80}$ while the remaining $2(40 \%)$ studies of associative learning reported no performance deficits. ${ }^{54,58}$ Bipolar disorder patients generally exhibited encoding-related hypo-activation in a network of inferior and middle frontal gyrus, ACC, dIPFC, superior parietal lobule, and insula regions in $80 \%$ of studies, independent of recall performance, ${ }^{54,58,79,80}$ or a combination of hypo-activation within nodes of this fronto-parietal network and dIPFC hyper-activity. ${ }^{54}$ During memory retrieval, remitted BD patients also showed primarily hypo-activation in the middle and inferior frontal gyrus and dIPFC, ${ }^{54,79}$ as well as of the hippocampus and parahippocampal gyrus. $^{24,54,79,80}$ Finally, one study also found less retrieval-related deactivation of $\mathrm{DMN}$ regions including the temporo-parietal junction. ${ }^{80}$

Learning and memory in unipolar disorder. In UD, 5 $(56 \%)$ studies found no differences on memory performance between $\mathrm{HC}$ and symptomatic or remitted UD. ${ }^{46,47,102,103,111}$ The last four (44\%) studies reported memory impairment in symptomatic (with and without psychotic features) and remitted patients. ${ }^{13,65,66,76}$

Encoding-related hippocampal and parahippocampal hypo-activation were observed in some studies of UD patients with recall deficits. ${ }^{13,65,66}$ Moreover, studies showing no behavioral differences generally failed to demonstrate any encoding- and retrieval-related hippocampal hypo-activity. ${ }^{46,102,103,111}$ Another consistent finding in UD patients was the absence of a "normal" association between greater hippocampal and parahippocampal activity during encoding and more subsequent retrieval success. ${ }^{13,46}$ Another common finding was that patients with poorer memory performance showed encoding-related hypo-activation in the middle and medial frontal gyrus and ACC. ${ }^{13,65,66}$ During memory retrieval, patients were found in several studies to hyperactivate prefrontal structures including the inferior frontal gyrus ${ }^{13,66,102,103}$ and hypo-activate the hippocampus and parahippocampal gyrus. ${ }^{24,65,76}$

\section{Attention}

Six studies examined the neural basis of attention: 3 in $\mathrm{BD}^{49,95,98}$ and 3 in UD. ${ }^{43,44,68}$

Attention in bipolar disorder. The 3 fMRI studies of manic and remitted BD patients focused on sustained attention, for which performance was impaired in 2 stu$\operatorname{dies}^{49,95}$ and comparable to HC in one study. ${ }^{98}$ Regardless of performance, BD patients exhibited replicated hyper-activation in limbic structures including the amygdala. ${ }^{49,98}$ Additionally, patients with performance impairments exhibited hypo-activation of cognitive control regions including the dlPFC, vlPFC, and parietal cortex. $^{49,95}$ In contrast, cognitively intact patients exhibited hyper-activation of the inferior frontal cortex, vlPFC, insula, and parietal regions. ${ }^{98}$

Attention in unipolar disorder. The 3 fMRI studies in symptomatic UD reported reduced sustained and selective attention performance, respectively. ${ }^{4,44,68}$ Two of 
these studies found that this deficit was accompanied by aberrant FC between fronto-parietal regions and occipital visual areas, resulting in decreased top-down modulation of early occipital processing. ${ }^{43,44}$ Korgaonkar et $a l^{68}$ also observed dlPFC hypo-activity during sustained attention. In contrast, no abnormal dlPFC activation was detected during a selective attention test despite impaired task performance. ${ }^{68}$ However, the study involved only region of interest (ROI) analysis, focusing on the dIPFC and dmPFC, and potential abnormalities in other cognitive control regions were thus not assessed.

\section{Implicit learning}

Two studies of symptomatic UD investigated implicit learning using a motor sequencing implicit learning task and a sequential finger-tapping task, respectively. ${ }^{53,77}$ Both found impaired implicit learning in patients, which was accompanied by dIPFC hypo-activity and impaired deactivation of the DMN. Patients also exhibited decreased FG between the hippocampus and PFC. ${ }^{53}$ In addition, one night of memory consolidation did not result in the "normal" reduction in task-related PFC and basal ganglia activation in UD patients. This could indicate disruption of the brain processes underlying implicit memory consolidation and thus less automation of responses in UD.

\section{Interim summary of cognitive impairment studies}

Taken together, the most consistent neural activity changes during working memory, executive skills, memory, and attention domains across BD and UD were abnormal activation of dlPFC, frontopolar, and parietal regions coupled with failure to deactivate the $\mathrm{DMN}$. Patients with BD generally exhibited PFC hypo-activation independent of performance levels, while UD patients generally displayed PFC hypo-activation of these structures when performance was impaired and hyperactivity in this region when performance was preserved. Another common marker of learning difficulties in mood disorders was encoding-related hypo-activation of middle frontal gyrus and ACC. A common phenomenon across the cognitive domains and diagnoses was also the abnormal FC within the PFC regions including the ACC and between PFC and subcortical/parietal regions. In contrast, hippocampal and parahippocampal hypo-activity was shown primarily during memory retrieval in BD and was not consistently observed in patients with UD. Instead, UD patients tended to lack the "normal" correlation between hippocampal engagement during encoding and subsequent retrieval success. Taken together, aberrant (hypo- and hyper-) activity (depending on the level of cognitive performance) in fronto-parietal cognitive control regions and failure to deactivate the
DMN may thus represent common fMRI biomarkers of cognitive impairments in mood disorders.

\section{Cognitive improvement}

Nine fMRI studies investigated the neural correlates of cognitive improvement in mood disorders, of which 5 focused on specific mood-independent cognitive improvement in response to candidate cognition treatments, ${ }^{22-25,123}$ and 4 investigated nonspecific cognitive improvement following symptom reduction ${ }^{106,124-126}$ (see Table 4).

\section{Specific treatment-related cognitive improvement}

The 5 studies that investigated specific treatment-related cognitive improvement focused on the effect of 3 different drug treatments [erythropoietin (EPO), ${ }^{22,23}$ lamotrigine, ${ }^{123}$ vortioxetine ${ }^{25}$ ] and one psychological intervention, cognitive remediation (CR) therapy. ${ }^{24}$ The treatment-associated changes were investigated on working memory in 4 studies and on learning and memory in 2 studies.

Working memory. Consistent findings regarding the neural correlates of treatment-related improvement of working memory were modulation of task-related activity in the cognitive control regions including the dlPFC and superior frontal gyrus ${ }^{23-25,123}$ and suppression of activity in DMN regions like the hippocampus. ${ }^{23,25}$ Specifically, Miskowiak et $a l^{23}$ showed in a randomized placebo-controlled controlled trial (RCT) that 8 weeks of treatment with erythropoietin (EPO) increased working memory capacity in UD and BD, which was accompanied by-and correlated with-enhanced task-related activity in the right superior frontal gyrus and deactivation of the left hippocampus. Similarly, Meusel et $a l^{24}$ found in an open-label uncontrolled study that CR therapy increased task-related activity in lateral PFC, medial frontal gyrus, superior temporal, and lateral parietal regions. As in the EPO trials, CR-related frontal and parietal activity increase correlated with improved working memory performance. It should be noted, however, that the CR-related working memory improvements did not reach statistical significance, and practice effects could not be excluded given the absence of a control group. The findings regarding the neural correlates of CR should therefore be interpreted with caution.

In contrast to the above-mentioned studies, Smith et $a l^{25}$ found that 2 weeks of treatment with the monoaminergic antidepressant vortioxetine reduced working memory-related dlPFC activity in remitted UD in the absence of changes in working memory performance. Notably, these patients displayed no objective impairment in working memory performance in 
TABLE 4. Studies of the neural underpinnings of cognitive improvement

\begin{tabular}{|c|c|c|c|c|c|c|c|c|}
\hline Author & Study design & Participants & Treatment & Duration & Test times & $\begin{array}{l}\text { fMRI paradigm } \\
\text { (cognitive domain) }\end{array}$ & Symptom change & Findings \\
\hline \multicolumn{9}{|l|}{$\begin{array}{l}\text { Neural correlates of } \\
\text { specific treatment- } \\
\text { related cognitive } \\
\text { improvement }\end{array}$} \\
\hline Miskowiak et al (2016) & $\begin{array}{l}\text { Randomized, double- } \\
\text { blind, placebo- } \\
\text { controlled, parallel- } \\
\text { group design }\end{array}$ & $\begin{array}{l}28 \text { moderately depressed } \\
\text { TRD (14 EPO) and } 34 \\
\text { partially remitted BD } \\
\text { (18 EPO) on } \\
\text { antidepressant/mood } \\
\text { stabilizing } \\
\text { medication. }\end{array}$ & EPO $><$ Saline & 8 weeks & $\begin{array}{l}\text { Baseline and week } \\
14\end{array}$ & $\begin{array}{l}\text { Explicit picture } \\
\text { encoding task } \\
\text { (learning and } \\
\text { memory) }\end{array}$ & $\begin{array}{l}\text { EPO did not affect } \\
\text { mood }\end{array}$ & $\begin{array}{l}\text { Performance: EPO } \downarrow \text { picture recall }><\text { saline. Activity: EPO Tencoding- } \\
\text { related bilateral dIPFC and left-side temporo-parietal response } \\
><\text { saline but did not affect encoding-related hippocampal activity. } \\
\text { Change in neural activity correlated with improvement of recall } \\
\text { performance. }\end{array}$ \\
\hline Meusel et al (2013) & $\begin{array}{l}\text { Open-label, } \\
\quad \text { noncontrolled design }\end{array}$ & $\begin{array}{l}28 \text { partially remitted } \mathrm{BD} / \\
\text { UD with concomitant } \\
\text { treatment and } 18 \mathrm{HC}\end{array}$ & $\begin{array}{l}\text { Cognitive } \\
\text { remediation }\end{array}$ & 10 weeks & $\begin{array}{l}\text { Baseline and week } \\
\quad 10\end{array}$ & $\begin{array}{l}\text { Adapted recollection } \\
\text { memory process } \\
\text { dissociation task } \\
\text { (learning and } \\
\text { memory) }\end{array}$ & $\begin{array}{l}\text { CR had no effect on } \\
\text { mood }\end{array}$ & $\begin{array}{l}\text { Performance: CR did not improve performance. Activity: CR } \mathbf{} \\
\text { recollection-related activation of right and left hippocampus. Changes } \\
\text { in hippocampal activity correlated with improvements in memory } \\
\text { performance. }\end{array}$ \\
\hline Haldane et al $(2008)^{123}$ & $\begin{array}{l}\text { Open-label, } \\
\quad \text { noncontrolled design }\end{array}$ & $\begin{array}{l}12 \text { stable BD } 1 \text { with no } \\
\text { concomitant } \\
\text { treatment ( } 8 \text { in final } \\
\text { analysis) }\end{array}$ & Lamotrigine & 12 weeks & $\begin{array}{c}\text { Baseline and } \\
12 \text { weeks }\end{array}$ & $\begin{array}{l}\text { N-back task (working } \\
\text { memory) }\end{array}$ & $\begin{array}{l}\text { Lamotrigine did not } \\
\text { affect mood }\end{array}$ & $\begin{array}{l}\text { Performance: No effect of lamotrigine. Activity: Lamotrigine } \boldsymbol{\uparrow} \text { activation } \\
\text { in the superior frontal, cingulate gyri, and left medial frontal gyrus. }\end{array}$ \\
\hline Smith et al (2017) & $\begin{array}{l}\text { Randomized, double- } \\
\text { blind, placebo- } \\
\text { controlled, parallel- } \\
\text { group design }\end{array}$ & $\begin{array}{l}48 \text { remitted UD with no } \\
\text { concomitant } \\
\text { treatment and } 48 \mathrm{HC}\end{array}$ & $\begin{array}{l}\text { Vortioxetine }>< \\
\quad \text { placebo }\end{array}$ & 2 weeks & $\begin{array}{l}\text { Baseline and } \\
2 \text { weeks (day } \\
\text { 12-14) }\end{array}$ & $\begin{array}{l}\text { N-back task (working } \\
\text { memory) }\end{array}$ & $\begin{array}{l}\text { Vortioxetine } \\
\text { improved self- } \\
\text { rated mood }\end{array}$ & $\begin{array}{l}\text { Performance: no effect of vortioxetine }><\text { placebo. Activity: vortioxetine } \\
\downarrow \text { WM-related activation in right dIPFC, left hippocampus, left } \\
\text { thalamus and right insular cortex). After adjustment in self-rated } \\
\text { mood, vortioxetine additionally } \downarrow \text { response in right insula, fusiform } \\
\text { gyrus, and lingual gyri. }\end{array}$ \\
\hline Miskowiak et al (2016) $)^{23}$ & $\begin{array}{l}\text { Randomized, double- } \\
\text { blind, placebo- } \\
\text { controlled, parallel- } \\
\text { group design }\end{array}$ & $\begin{array}{l}24 \text { moderately depressed } \\
\text { TRD (14 EPO) and } 32 \\
\text { partially remitted BD } \\
\text { (16 EPO) with } \\
\text { concomitant } \\
\text { treatment }\end{array}$ & EPO $><$ Saline & 8 weeks & $\begin{array}{l}\text { Baseline and week } \\
14\end{array}$ & $\begin{array}{l}\text { N-back task (working } \\
\text { memory) }\end{array}$ & $\begin{array}{l}\text { EPO did not affect } \\
\text { mood }\end{array}$ & $\begin{array}{l}\text { Performance: EPO } \uparrow \text { WM performance accuracy }><\text { saline. Activity: EPO } \\
\uparrow \text { right SFG and } \downarrow \text { left hippocampal activity (region of the DMN) }>< \\
\text { saline. Changes in neural activity correlated with improvement of } \\
\text { performance. }\end{array}$ \\
\hline Meusel et al (2013) & $\begin{array}{l}\text { Open-label, } \\
\text { noncontrolled design }\end{array}$ & $\begin{array}{l}23 \text { partially remitted } \mathrm{BD} / \\
\text { UD with concomitant } \\
\text { treatment and } 15 \mathrm{HC}\end{array}$ & $\begin{array}{l}\text { Cognitive } \\
\quad \text { remediation }\end{array}$ & 10 weeks & $\begin{array}{l}\text { Baseline and week } \\
10\end{array}$ & $\begin{array}{l}\text { N-back task (working } \\
\text { memory) }\end{array}$ & $\begin{array}{l}\text { CR had no effect on } \\
\text { mood }\end{array}$ & $\begin{array}{l}\text { Performance: CR did not improve performance. Activity: CR } \ \text { right and } \\
\text { left IPFC, right medial frontal, superior temporal, and lateral parietal } \\
\text { regions. Activation changes in frontal and parietal regions correlated } \\
\text { with changes in performance. }\end{array}$ \\
\hline \multicolumn{9}{|l|}{$\begin{array}{l}\text { Neural correlates of } \\
\text { nonspecific cognitive } \\
\text { improvement } \\
\text { following }\end{array}$} \\
\hline
\end{tabular}




\section{symptomatic \\ improvement}

Wagner et al (2010)

Prospective, naturalistic 20 depressed UD with

Citalopram (12) 6 weeks

Baseline and

Color-word Stroop

HDRS scores:

task (Executive

Reboxetine

controlled design benzodiazepines) and

(8)

skills)

$24 \pm 5 \rightarrow 9 \pm$

6; Citalopram:

$20 \mathrm{HC}$

$2+4 \rightarrow 8 \pm 6$

lifference between UD

activity after treatment (UD showed hyper-activation in fronto-parieto-

(

time for all UD: $\downarrow$ activity in left middle temporal lobe, right inferior parietal lobule, right ventrolateral prefrontal cortex and bilaterally in superior parietal lobe. Citalopram $\downarrow$ activity in right amygdala-

hippocampus complex.

\begin{tabular}{|c|c|c|c|c|c|c|c|c|}
\hline Kaladjian et al (2009) ${ }^{124}$ & $\begin{array}{l}\text { Case-control follow-up } \\
\text { study }\end{array}$ & 10 manic $\mathrm{BD}$ and $10 \mathrm{HC}$. & $\begin{array}{l}\text { Mood stabilizers, } \\
\text { antipsychotics, } \\
\text { or both }\end{array}$ & 143 days \pm 94 & $\begin{array}{l}\text { Baseline and } \\
\text { follow-up }\end{array}$ & $\begin{array}{l}\text { Go/no-go task } \\
\quad \text { (executive skills) }\end{array}$ & $\begin{array}{l}\text { Manic } \rightarrow \\
\quad \text { remission }\end{array}$ & $\begin{array}{l}\text { Performance: } \uparrow \text { from time } 1 \text { to time } 2 \text {. Activity: left amygdala the only } \\
\text { brain region to show a differential activation change over time } \\
\text { between BD and HC. BD } \downarrow \text { activation in left amygdala at } \mathrm{T} 2><\mathrm{T} 1 \text {. }\end{array}$ \\
\hline Walsh et al $(2007)^{125}$ & $\begin{array}{l}\text { Open-label, } \\
\quad \text { noncontrolled design }\end{array}$ & $\begin{array}{l}20 \text { depressed UD with no } \\
\text { concomitant } \\
\text { treatment and } 20 \mathrm{HC}\end{array}$ & $\begin{array}{l}\text { Fluoxetine } \\
\text { hydrochloride }\end{array}$ & 8 weeks & $\begin{array}{l}\text { Baseline and week } \\
2 \text { and } 8\end{array}$ & $\begin{array}{l}\text { N-back task (working } \\
\text { memory) }\end{array}$ & $\begin{array}{l}\text { HDRS scores: } \\
\qquad 21 \pm 2 \rightarrow 9 \pm 6\end{array}$ & $\begin{array}{l}\text { Performance: No effect of treatment (UD slower than HC). Activity: } \\
\text { Normalization of quadratic load-response in caudate nucleus and } \\
\text { thalamus in UD. }\end{array}$ \\
\hline Sankar et al (2017) & $\begin{array}{l}\text { Open-label, } \\
\text { noncontrolled design }\end{array}$ & $\begin{array}{l}23 \text { depressed UD with no } \\
\text { concomitant } \\
\text { treatment and } 22 \mathrm{HC}\end{array}$ & Duloxetine & 12 weeks & $\begin{array}{l}\text { Baseline, weeks } 1 \text {, } \\
8 \text {, and } 12\end{array}$ & $\begin{array}{l}\text { Delayed match-to- } \\
\text { sample/Sternberg } \\
\text { task (working } \\
\text { memory) }\end{array}$ & $\begin{array}{l}\text { HDRS scores: } \\
\qquad 22 \pm 3 \rightarrow 7 \pm 5\end{array}$ & $\begin{array}{l}\text { Performance: No effect of treatment (similar performance in UD and HC). } \\
\text { Activity: } \downarrow \text { encoding-related right precentral gyrus and left middle } \\
\text { temporal gyrus activity, } \downarrow \text { maintenance-related left inferior temporal } \\
\text { activity, } \downarrow \text { retrieval-related left inferior parietal activity, and } \downarrow \text { delayed } \\
\text { retrieval-related right precentral and cerebellum activity. }\end{array}$ \\
\hline
\end{tabular}

Abbreviations: EPO: Enythropoietin, TRD: Treatment resistant depression, HC: Healthy control, BD: Bipolar disorder, UD: Unipolar disorder, PFC: prefrontal cortex, dIPFC: dorsolateral PFC, IPFC: lateral PFC, SFG: superior frontal gyrus, rACC: Rostral anterior cingulate cortex, WM: Working memory, HDRS: Hamilton depression rating scale, YMRS: Young mania rating scale, BDI: Beck's depression inventory, SD: Standard deviation, T1: First time point, T2: Second time point, ><: Compared to. 
comparison with HC despite subjective cognitive complaints. $^{25}$ The reduced dlPFC activity in vortioxetine treated individuals was interpreted as increased cortical efficiency given the absence of overt change in these (cognitively intact) patients' working memory performance. Indeed, the previously noted distinction between efficiency and capacity is likely to explain the different direction of dIPFC change in response to vortioxetine vs. EPO treatment. Interestingly, vortioxetine also strengthened the deactivation of the hippocampus during working memory performance, similar to the effects of EPO. ${ }^{23}$

Finally, a small open-label, noncontrolled study of lamotrigine treatment in remitted BD patients revealed increased working memory-related activation over time in bilateral superior frontal and cingulate gyri and left medial frontal gyrus in the absence of changes in performance. ${ }^{123}$ However, it is difficult to determine whether this represents a beneficial effect on the neural activity associated with cognitive performance given (i) that the direction of activity change was opposite to the hypothesized (reduced) activity associated with greater efficiency as seen after vortioxetine treatment, (ii) the lack of associated cognitive improvement as seen after EPO treatment, and (iii) the within-group design with no control group, which could not exclude nonspecific effects of repeated testing and learning over time.

Learning and memory. Hippocampus and dIPFC were reported to underlie treatment-related improvements in the 2 studies of learning and memory. ${ }^{22,24}$ In the RCT by Miskowiak et $a l,{ }^{22}$ EPO treatment increased encodingrelated bilateral dIPFC and left-sided temporo-parietal response across $\mathrm{BD}$ and UD patients and improved subsequent recall performance. Importantly, the EPO-associated activity increase in dorsal PFC and temporo-parietal regions correlated with improvement of recall performance across the entire cohort, suggesting that this effect was mechanistically important. ${ }^{22}$ In contrast, no treatment-associated change in hippocampal response during memory encoding was observed. ${ }^{22}$ This contrasts with the finding by Meusel $e t a l^{24}$ of CR-related increase in hippocampus during retrieval. However, given the within-group design with no control group in the CR trial, the hippocampal activity increase over time could reflect nonspecific effects of repeated testing rather than specific effects of the intervention.

Cognitive improvement following symptom reduction

Four studies examined improvements in working memory and executive skills following reduction in mood symptoms, of which 3 studies were conducted in depressed UD patients ${ }^{106,125,126}$ and one in manic BD patients. $^{124}$
In general, cognitive improvement following symptom reduction was associated with decreased activation both within cognitive control and DMN regions. ${ }^{106,124,126}$ Indeed, Kaladjian et $a l^{124}$ found that improved cognitive performance in $\mathrm{BD}$ patients after transition from a manic to a remitted state was accompanied by decrease in left amygdala activation during an inhibitory control task. Similarly, Wagner et $a l^{106}$ found that reduction in depressive symptoms in UD patients after successful citalopram treatment was accompanied by decreased activity in the amygdala-hippocampus complex during color-word Stroop performance. Successful citalopram and reboxetine treatment of UD patients also attenuated pre-treatment hyper-activation of the fronto-parietotemporal network and rostral ACC during a cognitive control task. ${ }^{106}$ In contrast, task-related caudate nucleus and thalamus activity increase has also been observed in UD patients after reduction in depressive symptoms. ${ }^{125}$

Interim summary of cognitive improvement studies

Different pharmacological and psychological treatments that directly target cognition seem to specifically modulate dorsal PFC activity — with the direction of this activity change depending on the associated changes in performance levels-and to attenuate DMN hyperactivity. The observed opposite effects of EPO and vortioxetine on working memory-related dIPFC activity may be explained by the associated changes in capacity (ie, performance increase) or efficiency (with no associated behavioral change), respectively. Further, a common neural activity change observed after EPO and CR treatments was increase in task-related dlPFC activity. In contrast, encoding-related hippocampal activity was not modulated by EPO, and it is unclear whether the observed hippocampal activity increase after CR represented a treatment effect or nonspecific changes with repeated testing. Further, indirect cognitive improvement following symptom reduction was consistently accompanied by reduced limbic and DMN activity and reversal of pre-treatment fronto-parietal hyper-activity during task performance. This may represent reduced interference from hyper-active task-negative (limbic and DMN) regions after attenuation of mood symptoms and, consequently, less need for compensatory over-activation in cognition relevant regions.

\section{Discussion}

This systematic review examined the most consistent neural correlates for cognitive impairments and cognitive improvement in mood disorders to identify putative neurocircuitry-based targets for novel cognition treatments. We identified a total of 100 studies of the 
neuronal underpinnings of working memory, executive skills, learning and memory, attention, and implicit learning, respectively, and 9 studies of the neuronal changes associated with cognitive improvements. The most consistent findings regarding neural correlates for cognitive impairments across domains and diagnoses were aberrant (hypo- or hyper-) activity in medial and dorsal PFC cognitive control regions and parietal cortex, with the direction of this aberrant activity depending on cognitive performance levels as well as hyper-activity in the DMN and limbic regions. Candidate treatments that directly targeted cognition seemed to (i) specifically modulate dorsal PFC and temporo-parietal activity, with the direction of the activity change depending on whether it was accompanied by improved cognitive performance, and (ii) attenuate DMN hyper-activity. In contrast, indirect cognitive improvements following symptom reduction were commonly accompanied by attenuation of limbic hyperreactivity coupled with reversal of pre-treatment frontoparietal hyper-activity during cognitive performance.

\section{Putative biological targets for pro-cognitive treatments}

Remarkably, a few common brain regions were consistently identified as showing abnormal activity in UD and BD across a variety of fMRI paradigms tapping into different cognitive domains. Specifically, fMRI studies of working memory, executive skills, memory encoding, and sustained attention revealed reliable evidence for aberrant (predominantly hypo-) activity in dorsal PFC and fronto-polar regions as well as abnormal $\mathrm{FC}$ within the PFC and between the PFC, parietal, and limbic regions. Notably, the dorsal and lateral areas of the PFC are involved in a variety of "top-down" control processes that may be important across several cognitive domains, including active working memory maintenance and manipulation, attention control and -switching, impulse inhibition, and strategic encoding. ${ }^{19}$ This may explain the association between aberrant activity in these regions and impaired performance across diverse neurocognitive tests. Another consistent finding across fMRI studies of working memory, executive skills, and attention was reduced deactivation of the DMN and limbic structures during active task performance. This is in line with the hypothesis that cognitive impairments in mood disorders may exacerbated by a failure to suppress task-irrelevant neural activity associated with emotional reactivity, selffocus, and rumination. ${ }^{14,21}$ While abnormal hippocampal activity during memory retrieval was a reliable finding in BD patients, ${ }^{24,54,79,80}$ it was not consistently observed in UD patients. ${ }^{46,102,103,111}$ However, several studies found that UD patients failed to display the "normal" correlation between encoding-related hippocampal activation and subsequent retrieval success. This points to dysregulated encoding-related recruitment of the hippocampus rather than hippocampal hypo- or hyper-activity per se. Taken together, abnormal (predominantly hypo-) activity in dorsal and lateral PFC, aberrant PFC FC, and failure to suppress DMN activity emerged as the most consistent neural correlates of cognitive impairments across UD and BD and therefore represent the most promising biological targets for procognitive interventions.

\section{Efficiency vs. capacity: the importance of performance levels}

The discrepant findings regarding the direction of abnormal task-related activity in dorsal PFC (particularly in UD) and of the dorsal PFC activity change in response to pro-cognitive interventions ${ }^{22,23,25}$ are best explained in relation to patients' levels of cognitive performance. Specifically, dorsal PFC hyper-activity has been proposed to reflect reduced cortical efficiency, that is, the need for recruitment of more neural resources to maintain normal performance, ${ }^{127}$ whereas dorsal PFC hypoactivity is accompanied by reduced cognitive capacity, that is, performance decline when the task load exceeds individuals' cognitive capacity. ${ }^{127}$. Callicott et $a l^{127}$ proposed that the dorsal PFC hyper- and hypo-activity in patients can be explained by a leftward shift in the generally observed inverted U-shaped response curve between the cognitive task load and dorsal PFC activity. Specifically, neuropsychiatric patients may reach the peak BOLD response faster (ie, at a lower cognitive load) than HC, after which their dorsal PFC activity and associated performance success go down when the task load exceeds patients' cognitive capacity ${ }^{127}$ (for illustration, see our revised model based on Callicott et $a l^{127}$ in Figure 2). Indeed, we observed consistent evidence in this systematic review for dorsal PFC hypo-activity across $\mathrm{BD}$ and UD patients who showed impaired cognitive performance in comparison with $\mathrm{HC}$, whereas patients who maintained normal performance levels were commonly characterized by dorsal PFC hyper-activity. Also consistent with this model is the suggestion that frontopolar hypo-activation in $\mathrm{BD}$ results from the cognitive load exceeding patients' capacity to activate this region, which leads to deterioration of their task performance. ${ }^{63}$ Further, co-variation for performance levels in another study resulted in frontopolar hyper(rather than hypo-) activation in BD. ${ }^{29}$ Nevertheless, 2 studies failed to show such an association between hypoactivity and reduced cognitive capacity; instead they found dorsal PFG hyper-activation in patients with executive dysfunction. ${ }^{48,92}$ Given this, task-related PFC hyper-activity may also result from unsuccessful attempts to maintain normal task performance.

Consistent with the notion that abnormal dorsal PFC and DMN activity may be common biological targets for cognition treatments, the identified cognition trials 


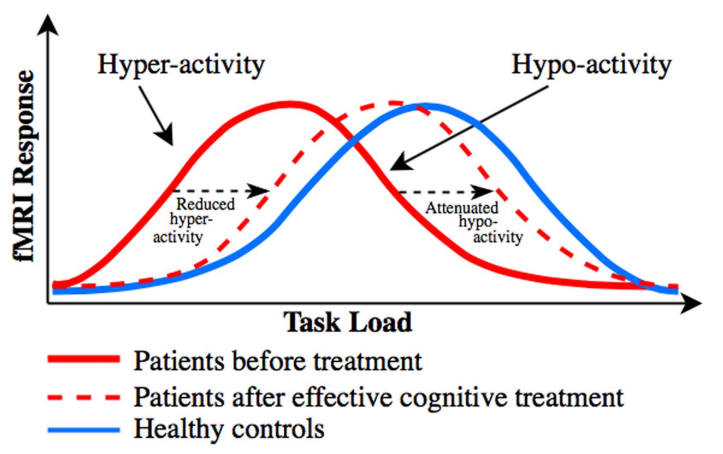

FIGURE 2. Putative distinct load-response curves, which may unify the discrepant findings regarding dorsal PFC activity change associated with cognitive impairment and cognitive improvement in mood disorders. Model revised from Callicott et al. ${ }^{127}$ Distinct inverted curves for the association between task load (task difficulty) and dorsal PFC activity in patients with neuropsychiatric disorders (red solid curve) and healthy controls (blue solid curve), and distinct changes in task-related dorsal PFC activity in response to treatments targeting cognition. Patients tend to display dorsal PFC hyper-activity in comparison with healthy controls when performance is maintained at medium task loads and dorsal PFC hypo-activity when performance declines at higher loads (where the task demand exceeds patients' cognitive capacity). We hypothesize that pro-cognitive treatments of patients shift the bell-shaped curve toward the right (ie, toward "normality"), as indicated with the red dotted curve. Depending on the cognitive task load and hence patients' performance levels, this rightward shift will be reflected by either $(A)$ reduction in pre-treatment dorsal PFC hyperactivity in (cognitively intact) patients who display no treatment-related change in performance (ie, increased cortical efficiency), such as seen after vortioxetine treatment, ${ }^{25}$ or (B) attenuation of pre-treatment dorsal PFC hypo-activity (ie, enhanced dorsal PFC response) in cognitively impaired patients who display treatment-related cognitive improvement (ie, enhanced cognitive capacity), as seen after enythropoietin ${ }^{22,23}$ and cognitive remediation treatments. ${ }^{24}$

revealed common treatment-related modulation of activity in these networks. In particular, EPO and CR increased task-related dorsal PFC and parietal activity, which correlated with increased recall performance. Further, modulation of working memory associated dIPFC activity was a common neural correlate of EPO and vortioxetine treatments, although the direction of this change differed between the interventions. We hypothesize that the apparent discrepancy regarding the direction of the dorsal PFC change can be explained by a common treatment-related rightward shift toward "normality" in the putative bell-shaped response curve between task load and dorsal PFC response ${ }^{127}$ (for illustration, see Figure 2). Depending on the cognitive task load and hence patients' performance levels, this rightward shift will be reflected by either (A) reduction in pre-treatment dorsal PFC hyper-activity in (cognitively intact) patients who display no treatment-related change in performance (ie, increased cortical efficiency), such as seen after vortioxetine treatment, ${ }^{25}$ or (B) attenuation of pre-treatment dorsal PFG hypo-activity (ie, enhanced dorsal PFC response) in cognitively impaired patients who display treatment-related cognitive improvement (ie, enhanced cognitive capacity), as seen after $\mathrm{EPO}^{22,23}$ and CR treatments ${ }^{24}$ (see Figure 2).

Remarkably, meta-analytical findings point to similar increase in task-related dlPFC and medial PFC activity as the most robust markers of cognitive improvements following CR treatments in schizophrenia. ${ }^{128}$ In contrast, CR-related activity change in other cognition-relevant regions, such as the hippocampus, was less consistent across schizophrenia trials. ${ }^{128}$ This is consistent with the lack of reliable treatment effects on encoding-related hippocampal activity in mood disorders. Together, these findings point to modulation of dorsal PFC and the DMN as the most promising surrogate marker of pro-cognitive effects of both pharmacological and cognitive treatments across several neuropsychiatric disorders.

Studies of indirect cognitive improvement following reduction in mood symptoms yielded a somewhat different pattern of neural changes: decrease in hyperactivity in limbic and DMN coupled with reversal of pretreatment fronto-parietal hyper-activity. Such indirect cognitive improvement could thus be mediated primarily by decreased interference from limbic and DMN hyperreactivity in parallel with patients' symptom reduction and consequent "relaxation" of the compensatory hyperactivity in cognitive control regions.

\section{Methodological challenges and opportunities}

A greater proportion of studies in UD than in BD patients displayed performance deficits on fMRI paradigms tapping into executive function, which contrasts with evidence for greater severity of cognitive deficits in BD. ${ }^{129}$ A likely explanation is that fMRI paradigms are generally not optimized for detection of deficits in cognitive performance but for detection of compensatory neural responses associated with intact cognitive performance. ${ }^{130}$ Nevertheless, the differential difficulty levels of the employed fMRI paradigms, together with patients' cognitive heterogeneity, may explain the common fronto-parietal hyper-activity in patients with intact cognitive performance and hypo-activity in those with compromised performance.

Functional MRI can provide a valuable dynamic measure of the treatment effects at a systems level in the brain, which may have better predictive validity than animal models. However, there are some fundamental limitations of the fMRI technique that must be considered in relation to its implementation in treatment development strategies targeting cognition. First, the reproducibility of the BOLD fMRI response is uncertain given inconsistent test-retest reliability across different assessment times in the same individuals. ${ }^{131}$ This limits the statistical power for detection of a treatment effect in 
fMRI studies with a repeated-measures design. Secondly, the fMRI BOLD response provides only an indirect measure of neural activity. This may be problematic for demonstrating neurocircuitry "target engagement" in response to treatments that influence global cerebral hemodynamic responses. ${ }^{131}$ Indeed, this turned out to be a problem in the EPO studies, since long-term EPO administration upregulates the level of red blood cells. Nevertheless, the problem was tackled by (i) postponing the post-treatment fMRI scan until the red blood cell counts had normalized (and verifying this with blood tests), and (ii) inclusion of a visual stimulation control task with no cognitive demands to examine whether there were any potential global (cognition-unrelated) differences in neural activity between EPO and saline groups. ${ }^{22,23}$ The golden standard approach would be to apply arterial spin labeling, which is an even more rigorous measure, to quantify and adjust for any potential physiological effects on global hemodynamic responses. Nevertheless, it may be argued that the fMRI BOLD response cannot provide a robust, reliable marker of treatment efficacy because the understanding of its biological basis is incomplete. Indeed, there is a lack of consensus in the field on whether treatment-related increase or decrease in fMRI BOLD is a marker of cognitive improvement. We propose a model that may explain these discrepant findings and become useful for interpretation of neuroimaging findings in future cognition trials (Figure 2). Specifically, the model involves consideration not only of the treatment-related change in dorsal PFC activity but also of the accompanying change in cognitive performance (or lack thereof) for the interpretation of the observed effects. Hence, assessment of treatment-related change in fMRI BOLD signal within key neurocircuitries together with change in cognition is a promising strategy for determining the functional relevance of any neural activity changes.

\section{Conclusion}

In conclusion, the most consistent neural underpinnings of cognitive impairments across cognitive domains and diagnoses were aberrant activity in the medial and dorsal PFC cognitive control regions and parietal cortex, with the direction of the aberrant activity depending on patients' cognitive performance levels. Another common finding was the failure to suppress DMN and limbic activity during cognitive performance. The findings from the cognition trials indicated that the most consistent biological targets for treatments with direct efficacy on cognition are (i) enhancement of activity in dorsal PFC cognitive control regions in patients with impaired cognitive performance (ie, increased capacity) or reduction of neural activity in these regions in patients with intact performance (ie, increased efficiency) (findings that may be reconciled with our proposed model for a rightward shift in the putative bell-shaped curve for the association between BOLD fMRI response and cognitive load; Figure 2), as well as (ii) suppression of activity in the DMN during cognitive performance. In contrast, indirect cognitive improvement following symptom reduction seemed to be mediated by decrease in limbic reactivity coupled with attenuation of fronto-parietal hyper-activity during cognitive performance. This review and integration of the findings in the field provide a first step toward a more unified understanding of the shared neural correlates of cognitive deficits in mood disorders and of treatment-associated cognitive improvements. These insights can provide a platform for studies assessing the predictive validity and reliability of treatment-related modulation of the dorsal PFC and DMN as surrogate markers for pro-cognitive effects. The perspective is the identification of a neurocircuitry biomarker model for pro-cognitive effects that can become a key tool to inform go/no-go decisions before the conduct of large-scale clinical efficacy trials in future treatment development programs.

\section{Disclosures}

Kamilla Miskowiak reports having received consultancy fees from Lundbeck and Allergan within the past 36 months.

\section{Supplementary material}

To view supplementary material for this article, please visit https://doi.org/10.1017/S1092852918001062

\section{REFERENCES:}

1. Whiteford HA, Degenhardt L, Rehm J, et al. Global burden of disease attributable to mental and substance use disorders: findings from the Global Burden of Disease Study 2010. Lancet. 2013;382(9904):1575-1586.

2. Bora E, Harrison BJ, Yucel M, Pantelis C. Cognitive impairment in euthymic major depressive disorder: a meta-analysis. Psychol Med. 2013;43(10):2017-2026.

3. Bourne C, Aydemir O, Balanza-Martinez V, et al. Neuropsychological testing of cognitive impairment in euthymic bipolar disorder: an individual patient data meta-analysis. Acta Psychiatr Scand. 2013;128(3):149-162.

4. Bonnin CM, Martinez-Aran A, Torrent C, et al. Clinical and neurocognitive predictors of functional outcome in bipolar euthymic patients: a long-term, follow-up study. J Affect Disord. 2010;121(1-2):156-160.

5. Torrent C, Martinez-Aran A, del Mar Bonnin C, et al. Long-term outcome of cognitive impairment in bipolar disorder. J Clin Psychiatry. 2012;73(7):e899-e905. 
6. Tse S, Chan S, Ng KL, Yatham LN. Meta-analysis of predictors of favorable employment outcomes among individuals with bipolar disorder. Bipolar Disord. 2014;16(3):217-229.

7. Olesen J, Gustavsson A, Svensson M, Wittchen HU, Jonsson B. The economic cost of brain disorders in Europe. Eur J Neurol. 2012;19 (1):155-162

8. Wyatt RJ, Henter I. An economic evaluation of manic-depressive illness-1991. Soc Psychiatry Psychiatr Epidemiol. 1995;30 (5):213-219.

9. Miskowiak KW, Carvalho AF, Vieta E, Kessing LV. Cognitive enhancement treatments for bipolar disorder: a systematic review and methodological recommendations. Eur Neuropsychopharmacol. 2016;26(10):1541-1561.

10. Miskowiak KW, Ott CV, Petersen JZ, Kessing LV. Systematic review of randomized controlled trials of candidate treatments for cognitive impairment in depression and methodological challenges in the field. Eur Neuropsychopharmacol. 2016;26 (12):1845-1867.

11. Miskowiak KW, Burdick KE, Martinez-Aran A, et al. Methodological recommendations for cognition trials in bipolar disorder by the International Society for Bipolar Disorders Targeting Cognition Task Force. Bipolar Disord. 2017;19(8): 614-626.

12. Carlson PJ, Singh JB, Jr Zarate CA, Drevets WC, Manji HK. Neural circuitry and neuroplasticity in mood disorders: insights for novel therapeutic targets. NeuroRx. 2006;3(1):22-41.

13. Dietsche B, Backes H, Stratmann M, Konrad C, Kircher T, Krug A. Altered neural function during episodic memory encoding and retrieval in major depression. Hum Brain Mapp. 2014;35 (9):4293-4302.

14. Fernandez-Corcuera P, Salvador R, Monte GC, et al. Bipolar depressed patients show both failure to activate and failure to deactivate during performance of a working memory task. J Affect Disord. 2013;148(2-3):170-178.

15. Frangou S, Kington J, Raymont V, Shergill SS. Examining ventral and dorsal prefrontal function in bipolar disorder: a functional magnetic resonance imaging study. Eur Psychiatry. 2008;23 (4):300-308.

16. Hamilton LS, Altshuler LL, Townsend J, et al. Alterations in functional activation in euthymic bipolar disorder and schizophrenia during a working memory task. Hum Brain Mapp. 2009;30(12):3958-3969.

17. Minzenberg MJ, Laird AR, Thelen S, Carter CS, Glahn DC. Metaanalysis of 41 functional neuroimaging studies of executive function in schizophrenia. Arch Gen Psychiatry. 2009;66(8):811-822.

18. Monks PJ, Thompson JM, Bullmore ET, et al. A functional MRI study of working memory task in euthymic bipolar disorder: evidence for task-specific dysfunction. Bipolar Disord. 2004;6 (6):550-564.

19. Ragland JD, Laird AR, Ranganath C, Blumenfeld RS, Gonzales SM, Glahn DC. Prefrontal activation deficits during episodic memory in schizophrenia. Amer J Psychiatry. 2009;166(8):863-874.

20. Townsend J, Bookheimer SY, Foland-Ross LC, Sugar CA, Altshuler LL. fMRI abnormalities in dorsolateral prefrontal cortex during a working memory task in manic, euthymic and depressed bipolar subjects. Psychiatry Res. 2010;182(1):22-29.

21. Sheline YI, Barch DM, Price JL, et al. The default mode network and self-referential processes in depression. Proc Natl Acad Sci US A. 2009;106(6):1942-1947.

22. Miskowiak KW, Macoveanu J, Vinberg M, et al. Effects of erythropoietin on memory-relevant neurocircuitry activity and recall in mood disorders. Acta Psychiatr Scand. 2016;134(3):249-259.

23. Miskowiak KW, Vinberg M, Glerup L, et al. Neural correlates of improved executive function following erythropoietin treatment in mood disorders. Psychol Med. 2016;46(8):1679-1691.
24. Meusel LA, Hall GB, Fougere P, McKinnon MC, MacQueen GM. Neural correlates of cognitive remediation in patients with mood disorders. Psychiatry Res. 2013;214(2):142-152.

25. Smith J, Browning M, Conen S, et al. Vortioxetine reduces BOLD signal during performance of the N-back working memory task: a randomised neuroimaging trial in remitted depressed patients and healthy controls. Mol Psychiatry. 2018;23(5):1127-1133.

26. Moher D, Liberati A, Tetzlaff J, Altman DG. Preferred reporting items for systematic reviews and meta-analyses: the PRISMA statement. PLoS Med. 2009;6(7):e1000097.

27. American Psychiatric Association. Diagnostic and Statistical Manual of Mental Disorders. 5th ed. Arlington, VA: American Psychiatric Publishing; 2013.

28. World Health Organization. The ICD-10 Classification of Mental and Behavioural Disorders: Clinical Descriptions and Diagnostic Guidelines. Vol. 1. Geneva: World Health Organization; 1992.

29. Adler CM, Holland SK, Schmithorst V, Tuchfarber MJ, Strakowski SM. Changes in neuronal activation in patients with bipolar disorder during performance of a working memory task. Bipolar Disord. 2004;6(6):540-549.

30. Ajilore O, Vizueta N, Walshaw P, Zhan L, Leow A, Altshuler LL. Connectome signatures of neurocognitive abnormalities in euthymic bipolar I disorder. J Psychiatr Res. 2015;68:37-44.

31. Allin MPG, Marshall N, Schulze K, et al. A functional MRI study of verbal fluency in adults with bipolar disorder and their unaffected relatives. Psychol Med. 2010;40(12):2025-2035.

32. Alonso-Lana S, Goikolea JM, Bonnin CM, et al. Structural and functional brain correlates of cognitive impairment in euthymic patients with bipolar disorder. PloS One. 2016;11(7):e0158867.

33. Alonso-Lana $\mathrm{S}$, Valenti M, Romaguera A, et al. Brain functional changes in first-degree relatives of patients with bipolar disorder: evidence for default mode network dysfunction. Psychol Med. 2016;46(12):2513-2521.

34. Barch DM, Sheline YI, Csernansky JG, Snyder AZ. Working memory and prefrontal cortex dysfunction: specificity to schizophrenia compared with major depression. Biol Psychiatry. $2003 ; \mathbf{5 3}(5): 376-384$.

35. Bartova L, Meyer BM, Diers K, et al. Reduced default mode network suppression during a working memory task in remitted major depression. J Psychiatr Res. 2015;64:9-18.

36. Blumberg HP, Leung HC, Skudlarski P, et al. A functional magnetic resonance imaging study of bipolar disorder: state- and trait-related dysfunction in ventral prefrontal cortices. Arch Gen Psychiatry. 2003;60(6):601-609.

37. Brandt CL, Eichele T, Melle I, et al. Working memory networks and activation patterns in schizophrenia and bipolar disorder: comparison with healthy controls. Br J Psychiatry. 2014;204 (4):290-298.

38. Brooks JO 3rd, Vizueta N, Penfold C, Townsend JD, Bookheimer SY, Altshuler LL.Prefrontal hypoactivation during working memory in bipolar II depression. Psychol Med. 2015;45(8):1731-1740.

39. Costafreda SG, Fu CH, Picchioni M, et al. Pattern of neural responses to verbal fluency shows diagnostic specificity for schizophrenia and bipolar disorder. BMC Psychiatry. 2011;11:18.

40. Crane NA, Jenkins LM, Dion C, et al. Comorbid anxiety increases cognitive control activation in major depressive disorder. Depress Anxiety. 2016;33(10):967-977.

41. Curtis VA, Thompson JM, Seal ML, et al. The nature of abnormal language processing in euthymic bipolar I disorder: evidence for a relationship between task demand and prefrontal function. Bipolar Disord. 2007;9(4):358-369.

42. Dell'Osso B, Cinnante C, Di Giorgio A, et al. Altered prefrontal cortex activity during working memory task in bipolar disorder: a functional magnetic resonance imaging study in euthymic bipolar I and II patients. J Affect Disord. 2015;184:116-122. 
43. Desseilles M, Balteau E, Sterpenich V, et al. Abnormal neural filtering of irrelevant visual information in depression. J Neurosci. 2009;29(5):1395-1403.

44. Desseilles M, Schwartz S, Dang-Vu TT, et al. Depression alters "top-down" visual attention: a dynamic causal modeling comparison between depressed and healthy subjects. Neuroimage. 2011;54(2):1662-1668.

45. Drapier D, Surguladze S, Marshall N, et al. Genetic liability for bipolar disorder is characterized by excess frontal activation in response to a working memory task. Biol Psychiatry. 2008;64 (6):513-520.

46. Fairhall SL, Sharma S, Magnusson J, Murphy B. Memory related dysregulation of hippocampal function in major depressive disorder. Biol Psychology. 2010;85(3):499-503.

47. Finkelmeyer A, Nilsson J, He J, et al. Altered hippocampal function in major depression despite intact structure and resting perfusion. Psychol Med. 2016;46(10):2157-2168.

48. Fitzgerald PB, Srithiran A, Benitez J, et al. An fMRI study of prefrontal brain activation during multiple tasks in patients with major depressive disorder. Hum Brain Mapp. 2008;29(4):490501.

49. Fleck DE, Eliassen JC, Durling M, et al. Functional MRI of sustained attention in bipolar mania. Mol Psychiatry. 2012;17 (3):325-336.

50. Frangou S. Brain structural and functional correlates of resilience to bipolar disorder. Front Hum Neurosci. 2011;5:184.

51. Frangou S. The Maudsley Bipolar Disorder Project. Epilepsia. 2005;46(Suppl 4):19-25.

52. Garrett A, Kelly R, Gomez R, Keller J, Schatzberg AF, Reiss AL. Aberrant brain activation during a working memory task in psychotic major depression. Am J Psychiatry. 2011;168(2):173-182.

53. Genzel L, Dresler M, Cornu M, et al. Medial prefrontalhippocampal connectivity and motor memory consolidation in depression and schizophrenia. Biol Psychiatry. 2015;77(2): 177-186.

54. Glahn DC, Robinson JL, Tordesillas-Gutierrez D, et al. Frontotemporal dysregulation in asymptomatic bipolar I patients: a paired associate functional MRI study. Hum Brain Mapp. 2010;31 (7):1041-1051.

55. Gruber O, Tost H, Henseler I, et al. Pathological amygdala activation during working memory performance: evidence for a pathophysiological trait marker in bipolar affective disorder. Hum Brain Mapp. 2010;31(1):115-125.

56. Gruber SA, Dahlgren MK, Sagar KA, et al. Decreased cingulate cortex activation during cognitive control processing in bipolar disorder. J Affect Disord. 2017;213:86-95.

57. Gruber SA, Rogowska J, Yurgelun-Todd DA. Decreased activation of the anterior cingulate in bipolar patients: an fMRI study. J Affect Disord. 2004;82(2):191-201.

58. Hall J, Whalley HC, Marwick K, et al. Hippocampal function in schizophrenia and bipolar disorder. Psychol Med. 2010;40(5): 761-770.

59. Hammar A, Neto E, Clemo L, Hjetland GJ, Hugdahl K, Elliott R. Striatal hypoactivation and cognitive slowing in patients with partially remitted and remitted major depression. Psych J. 2016;5 (3):191-205.

60. Harvey PO, Fossati P, Pochon JB, et al. Cognitive control and brain resources in major depression: an fMRI study using the n-back task. Neuroimage. 2005;26(3):860-869.

61. Hugdahl K, Rund BR, Lund A, et al. Brain activation measured with fMRI during a mental arithmetic task in schizophrenia and major depression. Am J Psychiatry. 2004;161(2):286-293.

62. Jamadar S, O'Neil KM, Pearlson GD, et al. Impairment in semantic retrieval is associated with symptoms in schizophrenia but not bipolar disorder. Biol Psychiatry. 2013;73(6):555-564.
63. Jogia J, Dima D, Kumari V, Frangou S. Frontopolar cortical inefficiency may underpin reward and working memory dysfunction in bipolar disorder. World J Biol Psychiatry. 2012;13 (8):605-615.

64. Joshi SH, Vizueta N, Foland-Ross L, et al. Relationships between altered functional magnetic resonance imaging activation and cortical thickness in patients with euthymic bipolar I disorder. Biol Psychiatry Cogn Neurosci Neuroimaging. 2016;1(6):507517.

65. Kassel MT, Rao JA, Walker SJ, et al. Decreased fronto-limbic activation and disrupted semantic-cued list learning in major depressive disorder. J Int Neuropsychol Soc. 2016;22(4):412-425.

66. Kelley R, Garrett A, Cohen J, et al. Altered brain function underlying verbal memory encoding and retrieval in psychotic major depression. Psychiatry Res. 2013;211(2):119-126.

67. Kikuchi T, Miller JM, Schneck N, et al. Neural responses to incongruency in a blocked-trial Stroop fMRI task in major depressive disorder. J Affect Disord. 2012;143(1-3):241-247.

68. Korgaonkar MS, Grieve SM, Etkin A, Koslow SH, Williams LM. Using standardized fMRI protocols to identify patterns of prefrontal circuit dysregulation that are common and specific to cognitive and emotional tasks in major depressive disorder: first wave results from the iSPOT-D study. Neuropsychopharmacology. $2013 ; 38(5): 863-871$.

69. Kronhaus DM, Lawrence NS, Williams AM, et al. Stroop performance in bipolar disorder: further evidence for abnormalities in the ventral prefrontal cortex. Bipolar Disord. 2006;8(1):28-39.

70. Lagopoulos J, Ivanovski B, Malhi GS. An event-related functional MRI study of working memory in euthymic bipolar disorder. $J$ Psychiatry Neurosci. 2007;32(3):174-184.

71. Le TM, Borghi JA, Kujawa AJ, Klein DN, Leung HC. Alterations in visual cortical activation and connectivity with prefrontal cortex during working memory updating in major depressive disorder. Neuroimage Clin. 2017;14:43-53.

72. Marchand WR, Lee JN, Thatcher GW, et al. A functional MRI study of a paced motor activation task to evaluate frontal-subcortical circuit function in bipolar depression. Psychiatry Res. 2007;155 (3):221-230.

73. Matsuo K, Glahn DC, Peluso MA, et al. Prefrontal hyperactivation during working memory task in untreated individuals with major depressive disorder. Mol Psychiatry. 2007;12(2):158-166.

74. McKenna BS, Sutherland AN, Legenkaya AP, Eyler LT. Abnormalities of brain response during encoding into verbal working memory among euthymic patients with bipolar disorder. Bipolar Disorders. 2014;16(3):289-299.

75. McKenna BS, Theilmann RJ, Sutherland AN, Eyler LT. Fusing functional MRI and diffusion tensor imaging measures of brain function and structure to predict working memory and processing speed performance among inter-episode bipolar patients. J Int Neuropsychol Soc. 2015;21(5):330-341.

76. Milne AM, MacQueen GM, Hall GB. Abnormal hippocampal activation in patients with extensive history of major depression: an fMRI study. J Psychiatry Neurosci. 2012;37(1):28-36.

77. Naismith SL, Lagopoulos J, Ward PB, Davey CG, Little C, Hickie IB. Fronto-striatal correlates of impaired implicit sequence learning in major depression: an fMRI study. J Affect Disord. 2010;125(1-3):256-261.

78. Norbury R, Godlewska B, Cowen PJ. When less is more: a functional magnetic resonance imaging study of verbal working memory in remitted depressed patients. Psychol Med. 2014;44 (6):1197-1203.

79. Oertel-Knochel V, Reinke B, Feddern R, et al. Verbal episodic memory deficits in remitted bipolar patients: a combined behavioural and fMRI study. J Affect Disord. 2013;150(2):430-440. 
80. Oertel-Knochel V, Reinke B, Feddern R, et al. Episodic memory impairments in bipolar disorder are associated with functional and structural brain changes. Bipolar Disord. 2014;16(8):830-845.

81. Palaniyappan L, Liddle PF. Diagnostic discontinuity in psychosis: a combined study of cortical gyrification and functional connectivity. Schizophr Bull. 2014;40(3):675-684.

82. Penfold C, Vizueta N, Townsend JD, Bookheimer SY, Altshuler LL. Frontal lobe hypoactivation in medication-free adults with bipolar II depression during response inhibition. Psychiatry Res. 2015;231(3):202-209.

83. Pompei F, Dima D, Rubia K, Kumari V, Frangou S. Dissociable functional connectivity changes during the Stroop task relating to risk, resilience and disease expression in bipolar disorder. Neuroimage. 2011;57(2):576-582.

84. Pompei F, Jogia J, Tatarelli R, et al. Familial and disease specific abnormalities in the neural correlates of the Stroop task in bipolar disorder. Neuroimage. 2011;56(3):1677-1684.

85. Rao JA, Kassel MT, Weldon AL, et al. The double burden of age and major depressive disorder on the cognitive control network. Psychol Aging. 2015;30(2):475-485.

86. Remijnse PL, van den Heuvel OA, Nielen MMA, et al. Cognitive inflexibility in obsessive-compulsive disorder and major depression is associated with distinct neural correlates. PloS one. 2013;8(4):e59600

87. Rive MM, Koeter MW, Veltman DJ, Schene AH, Ruhe HG. Visuospatial planning in unmedicated major depressive disorder and bipolar disorder: distinct and common neural correlates. Psychol Med. 2016;46(11):2313-2328.

88. Robinson JL, Bearden CE, Monkul ES, et al. Fronto-temporal dysregulation in remitted bipolar patients: an fMRI delayed-nonmatch-to-sample (DNMS) study. Bipolar Disord. 2009; 11(4):351-360

89. Rodriguez-Cano E, Sarro S, Monte GC, et al. Evidence for structural and functional abnormality in the subgenual anterior cingulate cortex in major depressive disorder. Psychol Med. 2014;44(15):3263-3273.

90. Rose EJ, Simonotto E, Ebmeier KP. Limbic over-activity in depression during preserved performance on the n-back task. Neuroimage. 2006;29(1):203-215.

91. Roth RM, Koven NS, Randolph JJ, et al. Functional magnetic resonance imaging of executive control in bipolar disorder. Neuroreport. 2006;17(11):1085-1089.

92. Ryan KA, Dawson EL, Kassel MT, et al. Shared dimensions of performance and activation dysfunction in cognitive control in females with mood disorders. Brain. 2015;138(5):1424-1434.

93. Schlösser RGM, Wagner G, Koch K, Dahnke R, Reichenbach JR, Sauer H. Fronto-cingulate effective connectivity in major depression: a study with fMRI and dynamic causal modeling. Neuroimage. 2008;43(3):645-655.

94. Schoning S, Zwitserlood P, Engelien A, et al. Working-memory fMRI reveals cingulate hyperactivation in euthymic major depression. Hum Brain Mapp. 2009;30(9):2746-2756.

95. Smucny J, Lesh TA, Newton K, Niendam T, Ragland JD, Carter CS. Levels of cognitive control: a functional magnetic resonance imagingbased test of an RDoC domain across bipolar disorder and schizophrenia. Neuropsychopharmacology. 2018;43(3):598-606.

96. Stegmayer K, Usher J, Trost S, et al. Disturbed cortico-amygdalar functional connectivity as pathophysiological correlate of working memory deficits in bipolar affective disorder. Eur Arch Psychiatry Clin Neurosci. 2015;265(4):303-311.

97. Strakowski SM, Adler CM, Cerullo M, et al. Magnetic resonance imaging brain activation in first-episode bipolar mania during a response inhibition task. Early Interv Psychiatry. 2008; 2(4):225-233.

98. Strakowski SM, Adler CM, Holland SK, Mills N, DelBello MP. A preliminary fMRI study of sustained attention in euthymic, unmedicated bipolar disorder. Neuropsychopharmacology. 2004;29(9):1734-1740.

99. Strakowski SM, Adler CM, Holland SK, Mills NP, DelBello MP, Eliassen JC. Abnormal fMRI brain activation in euthymic bipolar disorder patients during a counting Stroop interference task. Am J Psychiatry. 2005;162(9):1697-1705.

100. Taylor R, Theberge J, Williamson PC, Densmore M, Neufeld RW. ACC neuro-over-connectivity is associated with mathematically modeled additional encoding operations of schizophrenia Strooptask performance. Front Psychology. 2016;7:1295.

101. Thermenos HW, Goldstein JM, Milanovic SM, et al. An fMRI study of working memory in persons with bipolar disorder or at genetic risk for bipolar disorder. Am J Med Genet B Neuropsychiatr Genet. 2010;153B(1):120-131.

102. van Eijndhoven P, van Wingen G, Fernandez G, et al. Neural basis of recollection in first-episode major depression. Hum Brain Mapp. 2013;34(2):283-294.

103. van Eijndhoven P, van Wingen G, Fernandez G, et al. Amygdala responsivity related to memory of emotionally neutral stimuli constitutes a trait factor for depression. Neuroimage. 2011;54 (2):1677-1684.

104 van Tol MJ, van der Wee NJA, Demenescu LR, et al. Functional MRI correlates of visuospatial planning in out-patient depression and anxiety. Acta Psychiatr Scand. 2011;124(4):273-284.

105. Vasic N, Walter H, Sambataro F, Wolf RC. Aberrant functional connectivity of dorsolateral prefrontal and cingulate networks in patients with major depression during working memory processing. Psychol Med. 2009;39(6):977-987.

106. Wagner G, Koch K, Schachtzabel C, et al. Differential effects of serotonergic and noradrenergic antidepressants on brain activity during a cognitive control task and neurofunctional prediction of treatment outcome in patients with depression. J Psychiatry Neurosci. 2010;35(4):247-257.

107. Wagner G, Sinsel E, Sobanski T, et al. Cortical inefficiency in patients with unipolar depression: an event-related fMRI study with the Stroop task. Biol Psychiatry. 2006;59(10):958-965.

108. Walter H, Wolf RC, Spitzer M, Vasic N. Increased left prefrontal activation in patients with unipolar depression: an event-related, parametric, performance-controlled fMRI study. J Affect Disord. 2007;101(1-3):175-185.

109. Weathers J, Brotman MA, Deveney CM, et al. A developmental study on the neural circuitry mediating response flexibility in bipolar disorder. Psychiatry Res. 2013;214(1):56-65.

110. Welander-Vatn A, Jensen J, Otnaess MK, et al. The neural correlates of cognitive control in bipolar I disorder: an fMRI study of medial frontal cortex activation during a go/no-go task. Neurosci Lett. 2013;549:51-56.

111. Werner NS, Meindl T, Materne J, et al. Functional MRI study of memory-related brain regions in patients with depressive disorder. J Affect Disord. 2009;119(1-3):124-131.

112. Wessa M, Houenou J, Paillere-Martinot ML, et al. Fronto-striatal overactivation in euthymic bipolar patients during an emotional go/nogo task. Am J Psychiatry. 2007;164(4):638-646.

113. Wu G, Wang Y, Mwansisya TE, et al. Effective connectivity of the posterior cingulate and medial prefrontal cortices relates to working memory impairment in schizophrenic and bipolar patients. Schizophr Res. 2014;158(1-3):85-90.

114. Yoshimura Y, Okamoto Y, Onoda K, et al. Psychosocial functioning is correlated with activation in the anterior cingulate cortex and left lateral prefrontal cortex during a verbal fluency task in euthymic bipolar disorder: a preliminary fMRI study. Psychiatry Clin Neurosci. 2014;68(3):188-196.

115. Altshuler LL, Bookheimer SY, Townsend J, et al. Blunted activation in orbitofrontal cortex during mania: a functional magnetic resonance imaging study. Biol Psychiatry. 2005;58(10):763-769. 
116. Fleck DE, Kotwal R, Eliassen JC, et al. Preliminary evidence for increased frontosubcortical activation on a motor impulsivity task in mixed episode bipolar disorder. J Affect Disord. 2011;133(12):333-339.

117. Kaladjian A, Jeanningros R, Azorin JM, Nazarian B, Roth M, Mazzola-Pomietto P. Reduced brain activation in euthymic bipolar patients during response inhibition: an event-related fMRI study. Psychiatry Res. 2009;173(1):45-51.

118. Mazzola-Pomietto P, Kaladjian A, Azorin JM, Anton JL, Jeanningros R. Bilateral decrease in ventrolateral prefrontal cortex activation during motor response inhibition in mania. J Psychiatr Res. 2009;43(4):432-441.

119. McIntosh AM, Whalley HC, McKirdy J, et al. Prefrontal function and activation in bipolar disorder and schizophrenia. Am J Psychiatry. 2008;165(3):378-384.

120. Okada G, Okamoto Y, Morinobu S, Yamawaki S, Yokota N. Attenuated left prefrontal activation during a verbal fluency task in patients with depression. Neuropsychobiology. 2003;47(1):21-26.

121. Townsend JD, Bookheimer SY, Foland-Ross LC, et al. Deficits in inferior frontal cortex activation in euthymic bipolar disorder patients during a response inhibition task. Bipolar Disord. 2012;14 (4):442-450.

122. Welander-Vatn AS, Jensen J, Lycke C, et al. No altered dorsal anterior cingulate activation in bipolar II disorder patients during a go/no-go task: an fMRI study. Bipolar Disord. 2009;11(3):270-279.

123. Haldane M, Jogia J, Cobb A, Kozuch E, Kumari V, Frangou S. Changes in brain activation during working memory and facial recognition tasks in patients with bipolar disorder with lamotrigine monotherapy. Eur Neuropsychopharmacol. 2008;18 (1):48-54
124. Kaladjian A, Jeanningros R, Azorin JM, et al. Remission from mania is associated with a decrease in amygdala activation during motor response inhibition. Bipolar Disord. 2009;11 (5):530-538.

125. Walsh ND, Williams SC, Brammer MJ, et al. A longitudinal functional magnetic resonance imaging study of verbal working memory in depression after antidepressant therapy. Biol Psychiatry. 2007;62(11):1236-1243.

126. Sankar A, Adams TM, Costafreda SG, Marangell LB, Fu CH. Effects of antidepressant therapy on neural components of verbal working memory in depression. J Psychopharmacology. 2017;31 (9):1176-1183.

127. Callicott JH, Egan MF, Mattay VS, et al. Abnormal fMRI response of the dorsolateral prefrontal cortex in cognitively intact siblings of patients with schizophrenia. Am J Psychiatry. 2003;160(4):709719.

128. Ramsay IS, MacDonald AW 3rd. Brain correlates of cognitive remediation in schizophrenia: activation likelihood analysis shows preliminary evidence of neural target engagement. Schizophr Bull. 2015;41(6):1276-1284.

129. Gualtieri CT, Morgan DW. The frequency of cognitive impairment in patients with anxiety, depression, and bipolar disorder: an unaccounted source of variance in clinical trials. J Clin Psychiatry. 2008;69(7):1122-1130.

130. Price CJ, Friston KJ. Scanning patients with tasks they can perform. Hum Brain Mapp. 1999;8(2-3):102-108.

131. Zandbelt BB, Gladwin TE, Raemaekers M, et al. Within-subject variation in BOLD-fMRI signal changes across repeated measurements: quantification and implications for sample size. Neuroimage. 2008;42(1):196-206. 